\title{
Review
}

Irina Koryakina, Daria S. Kuznetsova, Dmitry A. Zuev, Valentin A. Milichko, Alexander S. Timin and Mikhail V. Zyuzin*

\section{Optically responsive delivery platforms: from the design considerations to biomedical applications}

https://doi.org/10.1515/nanoph-2019-0423

Received October 15, 2019; revised November 24, 2019; accepted December 10, 2019

\begin{abstract}
Drug carriers with intelligent functions are powerful therapeutic and diagnostic platforms in curing various diseases such as malignant neoplasms. These functions include the remote noninvasive activation of drug using physical impacts, e.g. light exposure. Combination of different therapeutic modalities (chemotherapy, photodynamic therapy, and so forth) with light-responsive carriers enables promising synergetic effect in tumour treatment. The main goal of this review article is to provide the state of the art on light-sensitive delivery systems with the identification of future directions and their implementation in tumour treatment. In particular, this article reviews the general information on the physical and chemical fundamental mechanisms of interaction between light and carrier systems (e.g. plasmonic and dielectric nanoparticles), the design of optically responsive drug carriers (plain and composite), and the mechanisms of light-driven controlled release of bioactive compounds in biological environment. The special focus is dedicated to the most recent advances in optically responsive bioinspired drug vehicles.
\end{abstract}

Keywords: light-responsive drug carriers; release mechanisms; plasmonic nanoparticles; resonant

*Corresponding author: Mikhail V. Zyuzin, Faculty of Physics and Engineering, ITMO University, Lomonosova 9, 191002 St. Petersburg, Russia, e-mail: mikhail.zyuzin@metalab.ifmo.ru. https://orcid.org/0000-0002-5364-2635

Irina Koryakina, Dmitry A. Zuev and Valentin A. Milichko: Faculty of Physics and Engineering, ITMO University, Lomonosova 9, 191002 St. Petersburg, Russia

Daria S. Kuznetsova: Privolzhsky Research Medical University, Institute of Experimental Oncology and Biomedical Technologies, Minin and Pozharsky Sq. 10/1, 603950 Nizhny Novgorod, Russia Alexander S. Timin: Research School of Chemical and Biomedical Engineering, National Research Tomsk Polytechnic University, Lenin Avenue 30, 634050 Tomsk, Russia dielectric nanoparticles; biomedical applications; tumour treatment.

\section{Introduction}

Drug delivery systems are designed to enable more effective drug administration, particularly, to deliver drugs to specific organs, to provide sufficient drug pharmacokinetics, and to improve solubility of drugs. Particulate delivery systems composed of organic and/or inorganic materials have proven their efficiency as drug carriers with different release mechanisms. Controlled drug release upon external and internal stimuli is one of the most important properties of drug carriers such as nanoparticles (NPs) of organic or inorganic nature, as well as bioinspired delivery platforms. Typically, the drug activation occurs in response to environmental triggering, for example, changes in $\mathrm{pH}$ [1] and ionic strength [2] (chemical stimuli), temperature [3] (physical stimulus), enzymatic degradation [4] (biological stimulus), and others [5]. However, researchers and medical professionals have aspired to focus on mechanisms for noninvasive drug activation using physical stimuli. Regarding the implementation of physical approaches for drug release: ultrasound [6], light [7], and magnetic field [8] can be used. Light is the most biocompatible external impact that is widely used to activate drug carriers and induce the cargo release [9]. Therefore, penetration depth of light is an important parameter for an effective noninvasive drug activation. Penetration of light irradiation with the wavelengths less than $700 \mathrm{~nm}$ [ultraviolet (UV) and visible] is rather limited to $1 \mathrm{~mm}$ due to the scattering and absorption from skin layers [10]. Application of the near-infrared (NIR) light irradiation (700-1000 nm) to trigger release of the bioactive molecules (drugs) inside biological objects allows to overcome this penetration barrier due to the lowest absorption coefficients of hemoglobin, lipids, and water in the NIR region [10]. 
There are two common mechanisms of light activation of bioactive compounds from the carriers: thermal effects and photochemical triggering (reassembly of organic carrier-structure upon light irradiation, which includes hydrophobicity-hydrophilicity transition or photocleavage reaction). Photothermally responsive drug carriers are able to adsorb light energy and convert it into heat. This heat further stimulates intracellular drug release from the carrier either by phase change mechanisms or by disruption of its structure. High temperatures (hyperthermia) also can reduce the cell viability; therefore, photothermal therapy (PTT) can be applied for such carriers along with the chemotherapy.

Another photoinduced mechanism involves either disruption of the hydrophobic-hydrophilic formulations with embedded photochromic moieties (e.g. micelles, liposomes [11]) or disassembly of the organic delivery platforms due to photocleavage reactions [12]. It is worth highlighting that the photochemical oxidation reaction is a special case of photocleavage mechanisms of drug release as reported by Bédard et al. [13]. Based on the design of light-responsive drug carriers, their interaction with light can vary, which in turn involves different mechanisms of drug activation. The photorelease profiles of bioactive compounds from light-sensitive drug carriers can be regulated via the adjustment of a number of parameters, such as light wavelength, light power intensity, duration of light exposure, and beam diameter [14, 15]. The important issue that should be taken into account is the stability of drug carriers in biological fluids. It is worth mentioning that after the first introduction of particulate drug carriers into biological fluids, organic compounds from these fluids tend to bind carriers' surface forming so-called protein corona [16]. This protein coating may undergo continuous adsorption and desorption, depending on the affinity of the surrounding proteins to the carrier's surface [17]. Protein corona can significantly change physicochemical properties of the carriers [18]. Moreover, it can affect particles light sensitivity [19]. For example, aggregated gold $\mathrm{Au})$ NPs can seriously induce the shift of plasmon band and therefore the efficiency of the light absorption [20].

For the design of light-responsive drug carries, various types of nanostructured materials based on plasmonic/ dielectric NPs [Au, silver (Ag), $\mathrm{Fe}_{2} \mathrm{O}_{3}, \mathrm{Fe}_{3} \mathrm{O}_{4}$, and others], photosensitizers (PSs), photochromic moieties [azobenzene (AZO), coumarin, spiropyran (SP), o-nitrobenzyl (NB), 4-bromo-7-hydroxycoumarin, and 2-diazo-1,2-naphthoquinone (DNQ)] have been considered. In addition, composite materials have gained interest as light-responsive components because they combine the merits of organic and inorganic components. As an example, micelle-, liposome-, and polymer-based materials modified with light-responsive parts can be fabricated [21-23]. Moreover, the inspiration from the biological side leads to the development of the cell-based delivery systems possessing light-addressable properties. Development of such delivery platforms usually involves the application of therapeutically relevant cells that can be naturally recruited in the tumour regions and deploy drugs upon laser irradiation. Various cell types have been reported as effective natural carriers, including mammalian cells [red blood cells (RBCs), neutrophils, leukocytes, phagocytes, stem cells (SCs), B lymphocytes, T lymphocytes, natural killer (NK) cells], bacteria, and viruses [24].

This review article aims to underline most recent advances of research on the light-sensitive drug delivery platforms. Special focus is dedicated to the consideration of the fundamental physical and chemical mechanisms describing light-matter interactions, which stimulate either conformational or thermal changes in the structure of the drug carriers. It further summarizes recent progress on the existing plain (individual plasmonic/dielectric materials) and composite (inorganic/organic nanostructured materials, as well as biomimetic platforms) drug delivery carriers, particularly on their design, drug loading/release possibilities, and biomedical applications (Figure 1).

\section{Fundamental principles of light interaction with plasmonic and dielectric NPs}

\subsection{Physical aspects of NPs' heating}

Resonant particulate nanophotonic structures are proven to be effective tools for precise optical heating of various systems at the nanoscale. This heating can be achieved by interaction of light with such plasmonic ( $\mathrm{Au}, \mathrm{Ag}$ ) and dielectric ( $\mathrm{Si}, \mathrm{Ge}$ ) nanometer-scale particles differently [25-29]. Indeed, different optical resonances (plasmonic and Mietype resonance) of these NPs allow electromagnetic field localisation with further conversion to heat energy by different paths and efficiencies. Moreover, these resonances can be simply tuned to biological transparency region in the NIR optical range by varying their physicochemical parameters such as size, shape, and composition. This enables to precisely control the heating process at the depth of several centimeters inside a tissue. In this section, we consider these fundamental mechanisms of conversion of 
Plain delivery systems
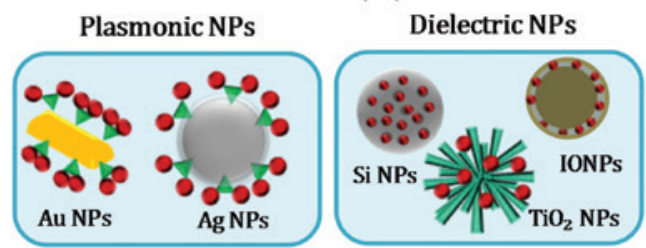

Remote controlled drug activation upon laser irradiation

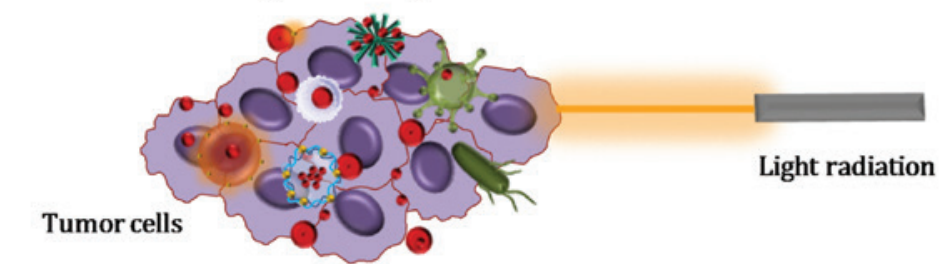

Drug release from light-sensitive delivery systems

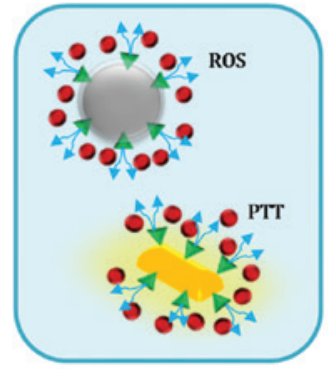

- Drug agents

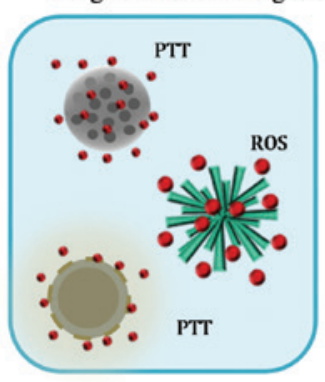

ROS

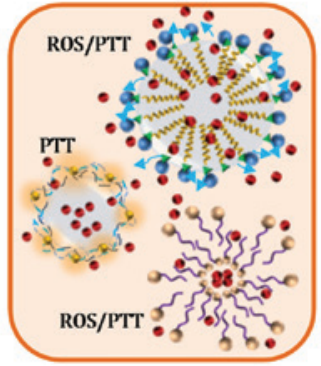

$\nabla$ Photosensitizer

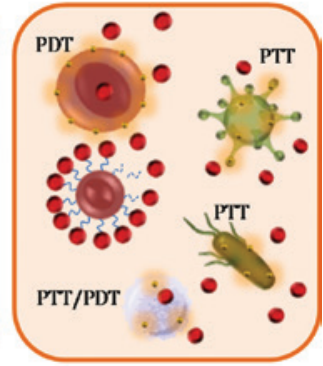

Heated Au NP

Figure 1: Schematic overview of light-sensitive delivery systems, as well as drug release mechanisms.

light energy to heat in plasmonic and dielectric NPs focusing on the nature of these processes and their dynamics.

\subsubsection{Plasmonic heating}

Photothermal therapy is one of the most remarkable examples of plasmonic heating application in biomedicine. This heating is based on the nature of plasmonic NPs' optical properties and arises from the resonant interaction of the electromagnetic field at certain frequencies with the oscillations of electrons (plasmons). These plasmons provide considerable enhancement of electric field near the NP surface as well as rise of optical absorption at the resonant frequency [20]. Generally, plasmonic heating can be described as the temperature accumulation and temperature distribution around the NP.

\subsubsection{Temperature accumulation}

As mentioned above, plasmonic NPs convert some part of this energy into heat under light irradiation, which is mainly related to absorption process. When considering the uniformly polarised spherical NP with the diameter, which is smaller than an excitation wavelength, this NP can be represented as an electromagnetic dipole (for larger spheres, Mie theory should be applied). In this case, the computation of the heat energy absorbed by plasmonic spherical NP strongly depends on the absorption crosssection $\sigma_{\text {abs }}$, which can be calculated as follows [26]:

$$
\sigma_{\text {abs }}=k \operatorname{Im}(\alpha)-\frac{k^{4}}{6 \pi}|\alpha|^{2}
$$

where $\alpha(\omega)=4 \pi R^{3} \frac{\varepsilon(\omega)-\varepsilon_{s}}{\varepsilon(\omega)+2 \varepsilon_{s}}$ is NP polarisability, $k$ is the angular wave number in the medium, $\varepsilon(\omega)$ is a complex relative permittivity of plasmonic NP, and $\varepsilon_{s}=n_{s}^{2}$ is a real relative permittivity of a medium around the spherical NP with a radius of $R$.

The plasmonic resonance takes place at the frequency $\omega$ when $\varepsilon(\omega) \approx-\varepsilon_{s}$. In turn, the absorption cross-section is related with scattering and extinction cross-sections $\left(\sigma_{\text {scat }}\right.$ and $\sigma_{\text {ext }}$ ) as follows [26]:

$$
\sigma_{\text {abs }}=\sigma_{\text {ext }}-\sigma_{\text {scat }}
$$

where $\sigma_{\text {scat }}=\frac{k^{4}}{6 \pi}|\alpha|^{2}$. 
The power absorbed by the NP $(Q)$ during irradiation is directly related with $\sigma_{\text {abs }}$ and intensity of the incident light $I$ as follows $[25,28]$ :

$$
Q=\sigma_{\text {abs }} I
$$

In case of complicated geometry, the power generated by the NP can be calculated through the heat power density $q(r)$ inside the NP with volume $V$ at the position $r$ as follows [25, 28]:

$$
Q=\int_{V} q(\boldsymbol{r}) d^{3} r=\int_{V} \frac{\omega}{2} \operatorname{Im}(\varepsilon(\omega)) \varepsilon_{0}|\boldsymbol{E}(\boldsymbol{r})|^{2} d^{3} r
$$

where $\boldsymbol{E}(\boldsymbol{r})$ is the electric field amplitude inside the NP, and $\varepsilon_{0}$ is dielectric permittivity.

It is obvious that $\sigma_{\text {abs }}$ plays an important role in the efficiency of conversion of light energy to heat. Table 1

Table 1: Comparison of the absorption cross-section for different plasmonic NPs.

\begin{tabular}{lrrrr}
\hline & Diameter of NPs $(\mathbf{n m})$ & $\boldsymbol{\omega}_{\boldsymbol{p}}(\mathbf{n m})$ & $\boldsymbol{\sigma}_{\text {abs }}\left(\mathbf{n m}^{2}\right)$ & Ref. \\
\hline $\mathrm{Au}$ & 10 & 532 & $\approx 5.0 \times 10^{1}$ & {$[30]$} \\
$\mathrm{Ag}$ & 10 & 400 & $\approx 0.5 \times 10^{3}$ & {$[31]$} \\
$\mathrm{Cu}$ & 10 & 310 & $\approx 2.5 \times 10^{1}$ & {$[32]$} \\
$\mathrm{Au}$ & 50 & 532 & $\approx 7.0 \times 10^{3}$ & {$[33]$} \\
$\mathrm{Ag}$ & 50 & 400 & $\approx 1.8 \times 10^{4}$ & {$[34]$} \\
$\mathrm{Pt}$ & 90 & 420 & $\approx 1.2 \times 10^{4}$ & {$[35]$} \\
$\mathrm{Au}$ & 90 & 532 & $\approx 2.0 \times 10^{3}$ & {$[33]$} \\
\hline
\end{tabular}

represents the estimated values of $\sigma_{\text {abs }}$ for different plasmonic materials, which can be used in PTT. Taking into account that $\mathrm{Au}$ is mostly nontoxic and biocompatible material [36], it allows to consider $\mathrm{Au}$ as the most suitable metal for plasmonic heating in nanomedicine. It is also important that the mechanisms of NP heating depend on laser modes, pulsed and continuous wave (CW). These processes are further discussed.

\subsubsection{Temperature distribution around the NP}

The good example illustrating the heating process of plasmonic NP is described in the work [37], where a spherical $\mathrm{Au} \mathrm{NP}$ with known radius $(R)$ immersed in water and uniformly illuminated at a resonant frequency is studied. In this case, the initial increase in the instantaneous temperature (moderate case) is considered; therefore, the changes in physicochemical properties of the material, mass transfer, cavitation, convection processes in liquid, oxidation of NP, possible molecular coatings, and others are not taken into account. Some of these examples can be found in several works [38-48].

It was established that the mode of laser irradiation (CW or pulsed) of plasmonic NP has a significant influence on the spatial extension of the temperature profile around the NP (Figure 2A), as well as temperature evolution (Figure 2B) $[28,37]$. This effect can be utilised to
A

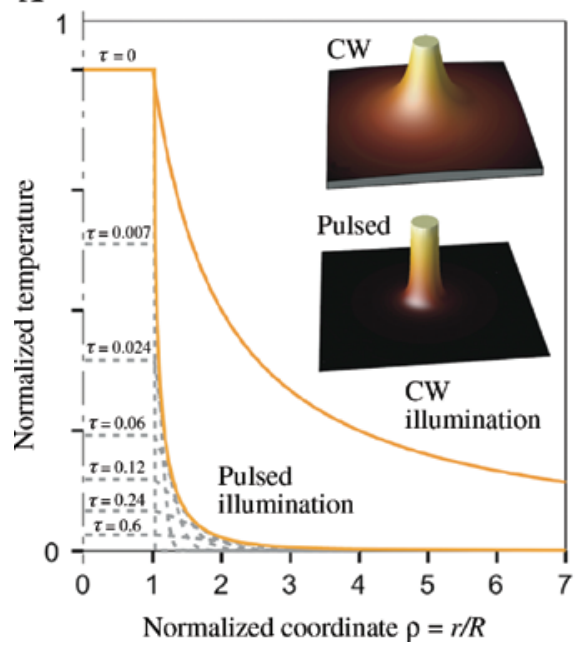

B

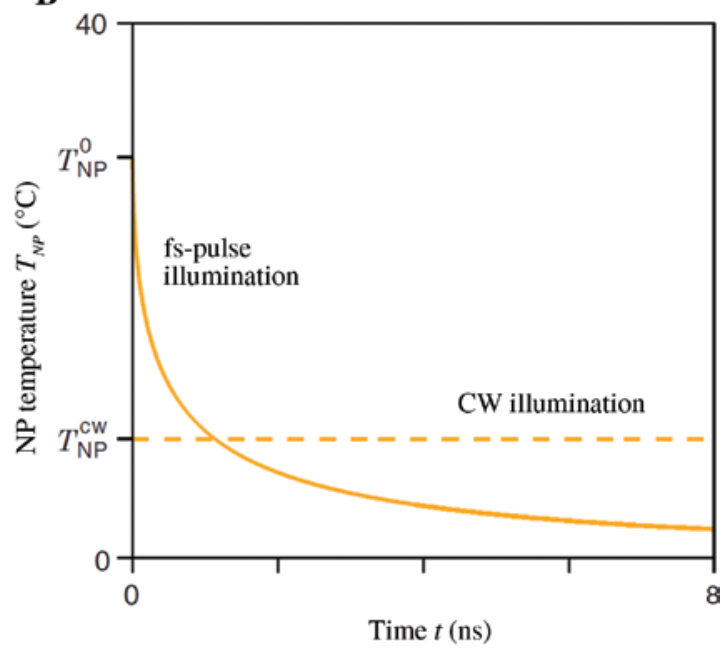

Figure 2: The modes of laser heating of Au NP in water.

(A) Spatial extension of the temperature profile in CW and pulsed modes of laser irradiation. The normalised time $\tau=t / \tau_{t r}$ is used in case of pulsed irradiation. Insets, 3D representation of the temperature profile around plasmonic NP under CW and single fs-laser pulse irradiation. (B) Comparison of the time-dependent temperature behaviour for the CW (dashed line) and single-pulse illumination (solid line) modes. Reprinted with permission from [28, 37]. Copyright 2013 by Wiley-VCH Verlag GmbH \& Co. KGaA, Weinheim; Copyright 2011 American Physical Society. 
control spatial region of heating and selectively start heatactivated chemical processes.

In the CW mode of irradiation and spherical shape of the NP, the steady-state temperature distribution $\delta T(r)$ outside the NP can be described as follows [28]:

$$
\delta T(r)=\delta T_{N P} \frac{R}{r}, r>R
$$

where $\delta T_{\mathrm{NP}}$ is the uniform temperature increase of the NP; $\delta T(r) \approx \delta T_{\mathrm{NP}}$ when $r<R$.

In turn, for the nanostructures with axial symmetry (rods, ellipsoids, discs, and tori), the temperature increase can be set as follows [49]:

$$
\delta T_{N P}=\frac{Q}{\beta 4 \pi \kappa_{s} R_{e q}}=\frac{\sigma_{\mathrm{abs}} I}{\beta 4 \pi \kappa_{s} R_{e q}}
$$

where $\beta$ is a dimensionless value introduced to correct geometry and equals 1 in case of sphere; $R_{e q}$ is the equivalent radius describing a sphere with the same volume as investigated for nonspherical NP; and $\kappa_{\mathrm{s}}$ is the thermal conductivity of the surrounding medium.

In the pulsed mode (picosecond and femtosecond scale), the interaction of laser radiation with a plasmonic NP can be generally described as step-by-step processes changing internal temperature of the NP and providing the temperature diffusion from heated NP to a surrounding media. These processes are schematically presented in Figure 3.

At first, the absorption of the laser energy by the free electron gas and its thermalisation (around 100 fs [37])

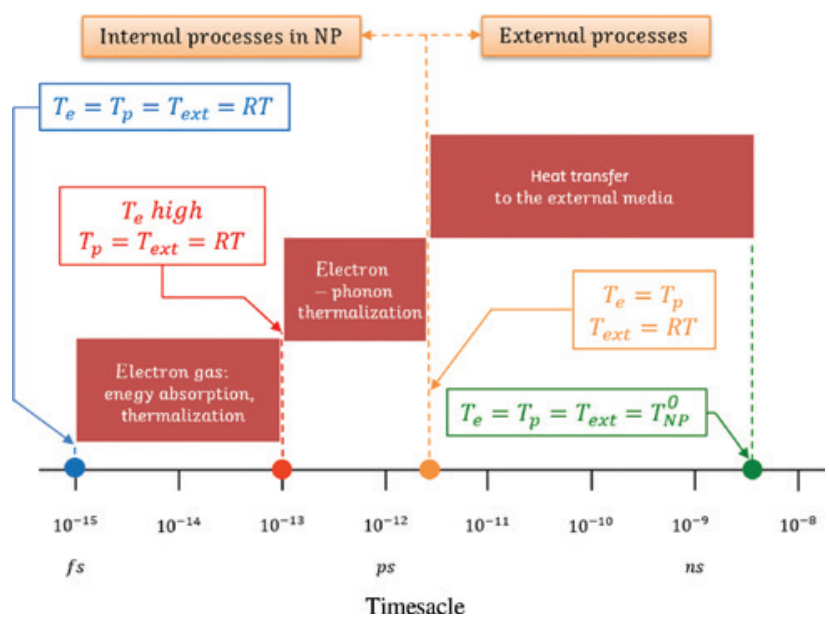

Figure 3: Schematic representation of processes taking place under pulsed ( $100 \mathrm{fs}$ ) laser irradiation of Au NPs.

The electronic temperature of the electronic gas $(T)$, temperature of the lattice $\left(T_{\mathrm{p}}\right)$, and surrounding media $\left(T_{\text {ext }}\right)$, as well as room temperature $(R T)$, are demonstrated on the scheme. take place. At this stage, the temperature of the ions of the metal lattice $T_{\mathrm{p}}$ remains unchanged (nonequilibrium conditions). Then electron-phonon interaction starts and provides internal equilibrium in the NP volume with uniform temperature of the electron gas $T_{\mathrm{e}}$ and metal lattice over a time scale of some units of ps $(\sim 1.7 \mathrm{ps}$ in case of Au NP) [37]. At this point, the temperature of a surrounding media $T_{\text {ext }}$ is still lower compared to the heated NP's temperature. The subsequent diffusion of heat from the NP to a media starts and lasts from the 10th of picoseconds to nanoseconds. To estimate the heat diffusion, the parameter of time $\tau_{t r}$ should be introduced [28],

$$
\tau_{t r} \sim L^{2} \frac{\rho c_{p}}{3 \kappa_{s}}
$$

where $L$ is the characteristic size of the NP (e.g. $R$ for a sphere), and $\rho$ and $c_{p}$ are the mass density of the NP and heat capacity at constant pressure, respectively.

It is worth mentioning that these processes are sizedependent. For example, electron-phonon thermalisation does not depend on the size of NPs larger than $5 \mathrm{~nm}$ $[37,50]$ and can overlap in time with the electron-phonon thermalisation process for NPs smaller than $20 \mathrm{~nm}[37,38]$.

The pulse repetition rate $f$ and laser fluence $F$ have an important impact on the heating process, and (3) can be introduced as follows $[28,37]$ :

$$
Q=\frac{\sigma_{\text {abs }}\langle I\rangle}{f}=\sigma_{\text {abs }} F
$$

where $I$ is average irradiance. In this case, the value of the initial temperature of the NP can be described as follows [37]:

$$
T_{\mathrm{NP}}^{0}=\frac{\sigma_{\mathrm{abs}} F}{V \rho_{\mathrm{Au}} c_{\mathrm{Au}}}
$$

where $V$ is the volume of the NP.

It is important to note that the pulse repetition rate can be tuned to provide quasi-CW heating mode. In this case, heat will be delivered into the NP so fast that there will be no time for the NP cooling $\left(f \gtrsim 1 / \tau_{t r}\right)$. Pulse duration and its ratio to $\tau_{t r}$ are other parameters, which have significant influence on the heating/cooling dynamics in the pulsed mode [28]. When the small NP (less than $100 \mathrm{~nm}$ ) is irradiated by the picosecond or femtosecond laser pulses, the interaction of the laser pulse with the NP can be observed in Figure 3, and the time of heating equals $\tau_{t r}$ (due to magnitude). Otherwise, under nanosecond pulses, the duration of heating is determined only by the duration of the laser pulse (the processes in Figure 3 time overlap) $[28,37]$. 
An example of the different heating processes is shown in the recent work of Halas and coworkers, where authors experimentally revealed the variations in heating mechanisms under the investigation of light-triggered deoxyribonucleic acid (DNA) release from $\mathrm{Au}$ NPs [15]. Indeed, CW laser illumination drives the photothermal release of dehybridised single-stranded DNA, whereas femtosecond excitation results in negligible local heating followed by DNA release by breaking of the thiol bond. The authors underlined the importance of application of such NPs irradiated by different laser modes in the development of NIR-triggered gene or drug delivery.

\subsubsection{Heating of dielectric NPs}

Another approach of the nanoscale heating is based on the utilisation of resonant NP made of high refractive index materials [26, 29, 51]. Initially, such NPs were not considered as effective heating tools. Indeed, metal NPs can provide high temperatures due to significant ohmic losses, which makes them very efficient for optically induced heating. The main drawback of heating in metal NPs is that this process is poorly controlled. Moreover, it can harm the nanostructure itself or/and lead to undesirable chemical reactions in surrounding media [29]. In contrast, high-index (dielectric) materials possess low optical losses (no charged carriers) and therefore at the first sight cannot provide significant optically induced heating. However, recently it has been demonstrated that it is possible to achieve high light-to-heat conversion in these systems [26]. In particular, magnetic quadrupole resonances in silicon (Si) NPs have been employed to generate highly efficient optical heating.
To compare the heating process in a resonant NP (metal or dielectric), (3) can be introduced [26],

$$
Q \sim \sigma F^{2} V_{\text {eff }}
$$

where $\sigma=\varepsilon_{0} \omega \operatorname{Im}(\varepsilon)$ is electric conductivity, $\mathrm{F}=\left\langle|E|^{2}\right\rangle /\left|E_{0}\right|^{2}$ is spatially averaged field enhancement factor, and $V_{\text {eff }}$ is the effective mode volume inside the NP.

Therefore, these three components contribute to NP heating. Taking into account optical losses, the impact of the first two components $\left(\sigma F^{2}\right)$ can be considered, including the radiative and ohmic components. At the resonant frequency,

$$
\sigma F^{2} \sim \frac{\gamma_{\text {Ohmic }}}{\left(\gamma_{\text {Ohmic }}+\gamma_{\text {rad }}\right)^{2}}
$$

In this case, the maximum value of $\sigma F^{2}$ can be achieved when $\gamma_{\mathrm{Ohmic}} \approx \gamma_{\mathrm{rad}}$ [28]. This requirement is satisfied for small metallic and big dielectric NPs (Figure 4).

According to (10), the effective mode volume inside the NP $V_{\text {eff }}$ has also influence on the NP heating and can be calculated as follows [26]:

$$
\begin{aligned}
& V_{\text {eff }}=\pi D^{2} \delta(\text { plasmonic NP }), \\
& V_{\text {eff }} \approx \pi D^{3} / 6(\text { dielectric NP }),
\end{aligned}
$$

where $\delta$ is a skin depth (less than $20 \mathrm{~nm}$ in the visible range).

Thus, increase in the NP size for efficient opticalinduced heating is more reasonable in case of resonant dielectric NPs, which, at appropriate sizes, can provide comparable heating with plasmonic ones and sometimes even exceed it (Figure 5A).

Another interesting feature of dielectric materials is that many of them demonstrate Raman signal, which is

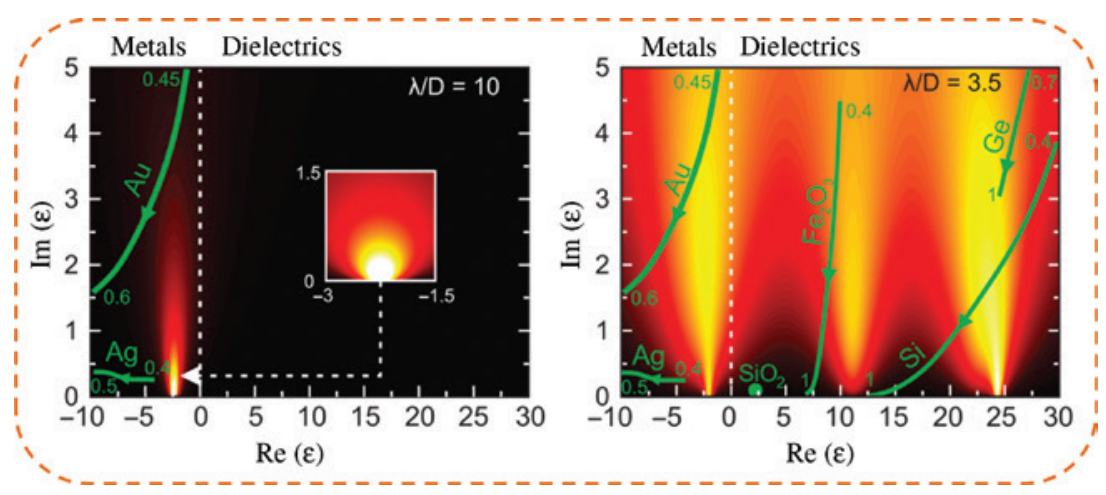

Figure 4: Calculated resonant heating maps for a spherical NP with diameter $D$ in the air for different real and imaginary parts of permittivity made for various ratios of wavelength $\lambda$ to $D$.

In the calculations, $\lambda$ is fixed, green lines depict dispersion for materials studied, and direction of arrows shows the wavelength increase (in micrometers). Reprinted with permission from [26]. Copyright 2017 American Chemical Society. 


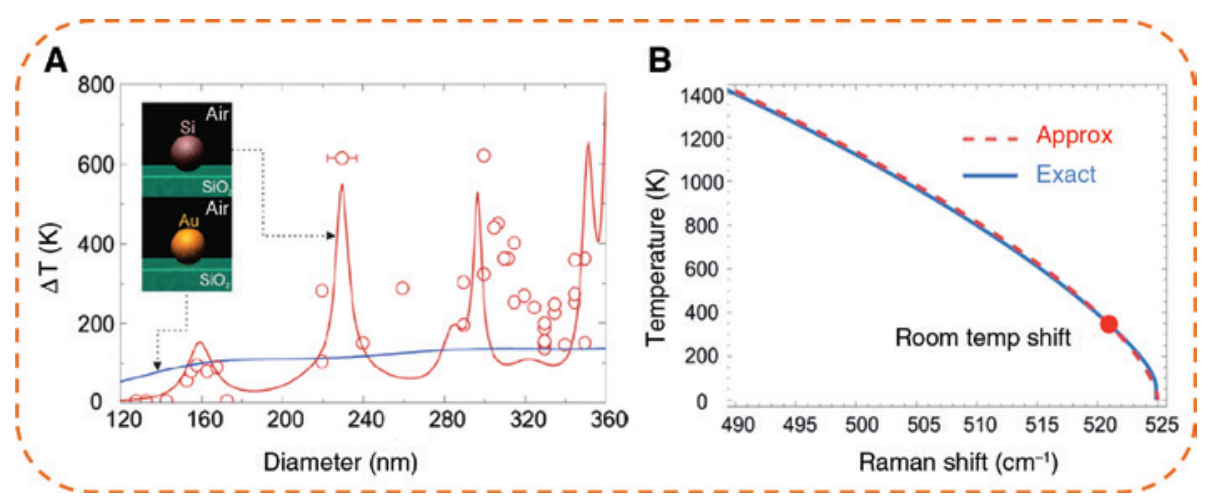

Figure 5: Optically induced heating and thermometry in high-refractive index NPs.

(A) Comparison of temperatures achieved in plasmonic and dielectric NPs. The results of theory (solid lines) and experiment (circles) are represented. Heating conditions, $\lambda=633 \mathrm{~nm}, I_{0}=2 \mathrm{~mW} / \mu \mathrm{m}^{2}$. (B) Results of calculation of the temperature-dependent Raman shift on the temperature for Si NP from (13) (solid line) and its approximation (dotted line). Reprinted with permission from [26, 52]. Copyright 2017 American Chemical Society; Copyright 2017 by Wiley-VCH Verlag GmbH \& Co. KGaA, Weinheim.

not observed in plasmonic materials. This effect can be employed for developing the all-in-one system based on resonant dielectric NPs, which unifies optically induced heating with the thermometry at the nanoscale. Moreover, it has been demonstrated that resonant properties of such NPs can also be applied for the enhancement of Raman signal (e.g. 140 times at the magnetic dipole resonance in spherical Si NPs) [53].

The dependence of the Raman line shift $\Omega$ on dielectric NP temperature can be considered on the example of crystalline Si NP. The temperature can be calculated as follows [26, 52]:

$$
\Omega(T)=\Omega_{0}+A\left(1+\frac{2}{e^{x}-1}\right)+B\left(1+\frac{3}{e^{y}-1}+\frac{3}{\left(e^{y}-1\right)^{2}}\right)
$$

where $\Omega_{0}=528 \mathrm{~cm}^{-1}, x=\frac{\hbar \Omega_{0}}{2 k_{B} T}, y=\frac{\hbar \Omega_{0}}{3 k_{B} T}, A=-2.96 \mathrm{~cm}^{-1}$, $B=-0.174 \mathrm{~cm}^{-1}$ for crystalline $\mathrm{Si}$, and $k_{B}$ is the Boltzmann constant.

The plotted temperature dependence of Raman signal shift can be approximated in Figure 5B with simple analytical equation [52],

$$
T_{\text {appr }}=140 \times(525-\Omega)^{0.65}
$$

Thus, the opportunity of the Raman nanothermometry combined with the high temperatures, which can be achieved at optical resonances in dielectric NP under optically induced heating, makes them an attractive platform for a wide range of applications [54-56]. The optical thermometry at the nanoscale available in these materials can be effectively used in the field of drug delivery to precisely control the temperature of drug release inside cell and avoid undesirable overheating. However, it is worth to mention that under high values of laser fluence or temperatures, thermooptical nonlinear effects can change refractive index and thermal conductivity of heated materials.

Compared to plasmonic NPs, the key difference of heating of dielectric NPs is the ability to heat them to higher temperatures up to $1000 \mathrm{~K}$ and more with less pumping laser intensity [52, 54]. This opens up the possibility of utilisation of such NPs for drug delivery without phototoxicity [51], as well as for high-temperature photochemistry and catalysis at the nanometer scale. Indeed, Si quantum dots were proven to be effective nanosystems for applications in cell biology and medicine [57]. In turn, application of optically resonant high-refractive index nanoparticles (e.g. $\mathrm{Si}$, $\mathrm{Ge}, \mathrm{Fe}_{2} \mathrm{O}_{3}$, etc.) makes it possible to add another opportunity related with optical-induced heating and control of chemical reactions in different systems at the nanoscale. For example, Zograf et al. employed resonant dielectric NPs to induce release of antitumour drug from biocompatible polymer microcontainers upon pulsed NIR-laser irradiation with less applied power density. Noninvasive intracellular release was probed on two cell types, carcinoma cells and SCs. The developed systems based on polymer containers decorated with dielectric resonant particles were found to have a high potential as drug delivery platforms [51].

\subsection{Chemical aspects of light-NP interaction}

The electron ejection from the metal surface by light, so-called photoelectric effect, was discovered by Heinrich Hertz [58] in 1887 during the study of the interaction between UV light and metals. Afterwards, Albert Einstein provided the explanation of this effect in 1905 by the following statement [59]: light consists of a set of photons 
with a discrete energy allowing electron ejection from a metal surface with a certain threshold, i.e. a minimum photon energy equals the metal's work function. These discoveries induced the quantum revolution and stimulated research in different fundamental directions, including complex physical (discussed above) and chemical processes that can be excited by light.

In this section, we discuss the process of light-NP interaction from the chemical point of view. Figure 6 illustrates photodissociation, decomposition, desorption, and quenching of chemical environment adsorbed to the surface of the NPs, as well as phototherapy. Interestingly, regardless of the metallic or dielectric nature of the NPs, these processes are the result of heating the NPs by light and light-induced transfer of charges or energy (Figure 6).

As discussed above, the light-induced heating of metallic (plasmonic) and dielectric NPs includes different mechanisms and involves a cascade of complex processes [60]. In the former case, the excited plasmons can relax within tens of femtoseconds by nonradiative way and excite energetic charge carriers (electrons and holes). Afterwards, the relaxation from non-Fermi to Fermi electron distribution occurs through electron-electron scattering. Cooling of this electronic gas takes picoseconds due to electron-phonon interaction with the following heat dissipation from the NP to surrounding media through phonon-phonon scattering. In case of dielectric NPs, after excitation of the

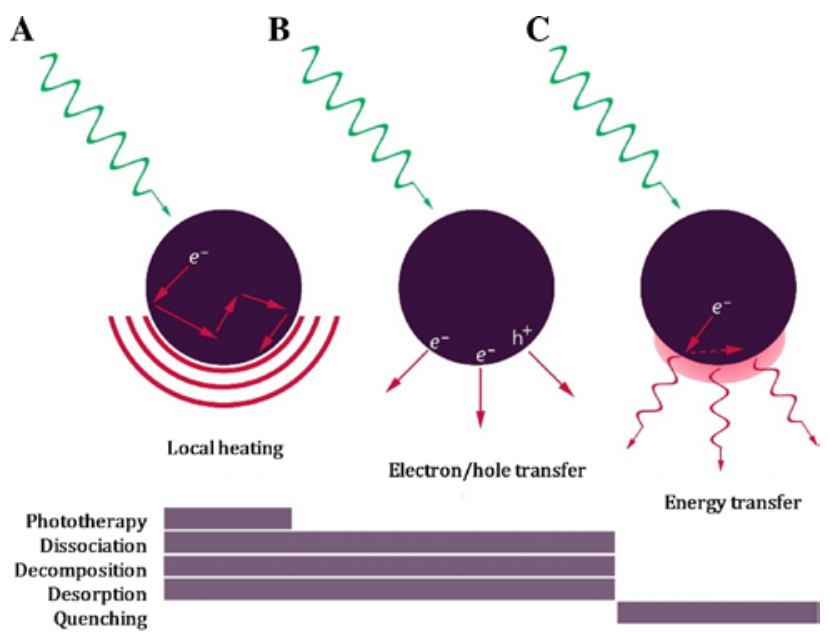

Figure 6: Schematic illustration of the light-NP interaction. (A) Local heating of NPs and adsorbates, (B) electron and/or hole transfer to adsorbates, and (C) energy transfer from/to NPs. These three mechanisms allow observing chemical transformation of surroundings including photodissociation, photodecomposition, photodesorption, and other. The green arrows represent the incoming light, while the red arrows correspond to electron relaxation with the loss of its energy and re-emission of the light. The heat distribution is represented as hemispheres. charged carriers from the valence to conduction band, the relaxation of electrons occurs also through electron-phonon interaction with following heat dissipation from the NP surface. As a result, the energy transfer from photons to thermal energy of NP adsorbates (nuclear motion of atoms) takes from picoseconds to microseconds and activates the chemical bonds and chemical transformations with the following consequences.

\subsubsection{Desorption (photons to thermal energy)}

In some cases, the light-induced heating of the NPs with different adsorbates is applied for remote on-demand optical delivery/release of bioactive compounds with high spatial resolution [61]. Here we discuss the important issues of desorption from the chemical point of view - the unbinding between adsorbates and NPs. It should be noted that desorption of organic compounds from the NP surface requires different energy depending on bonding. On the one hand, noncovalent bonding as hybridisation (hydrogen bonds, which, for example, results in formation of double-stranded DNA) [15] or electrostatic interaction (as an example, the interaction between cationic polymers and negatively charged single-stranded and double-stranded nucleic acids) requires a little (up to several $\mathrm{meV}$ ) thermal or electronic energy for desorption [62]. This interaction is also important for protein corona formation around NPs $[16,54]$. On the other hand, the covalent bonding is generally achieved by coupling with amino acid residues as $-\mathrm{NH}_{2},-\mathrm{SH}$, and - $\mathrm{COOH}$. In the case of covalent bonding, the dissociation of the bonds as thiol group, amine-carboxylate coupling, and complex of azide-tagged NP and alkyne-tagged adsorbate [63] requires much higher energy at least $0.1 \mathrm{eV}$ by heating or electronic injection $[15,62,64]$.

\subsubsection{Photodissociation and decomposition (photons to thermal energy)}

The catalytic reactions such as ammonia synthesis, hydrocarbon reforming, oxidation, hydrogenation, and others are generally triggered by heating at relatively high temperatures $\left(500^{\circ} \mathrm{C}\right.$ or greater). This process involves the excitation of phonons of the NP and their coupling to vibrational modes of the adsorbates [65]. For instance, heating of Au NPs dispersed in water-ethanol mixture results in decomposition of ethanol to $\mathrm{CO}_{2}, \mathrm{H}_{2}$ and $\mathrm{CO}$, whereas dicumyl peroxide can be decomposed by light on the surface of Au NP into 2-phenyl-2-propanol and acetophenone $[66,67]$. 


\subsubsection{Phototherapy (photons to thermal energy)}

The electron-phonon scattering in plasmonic NPs can result in significant heating to the melting temperature of NPs. As discussed, the tuning of the NP shape and size can shift the plasmon resonance to NIR range, where the light absorption of tissues is limited. In case of therapy with plasmonic NPs, the illumination results in local efficient heating of these NPs with following cellular hyperthermia, cell death, and tumour remission, as well as treatment of epithelial carcinoma SK-BR-3, photothermal destruction of malignant squamous cell, and others [6870]. Moreover, owing to local heating, plasmonic NPs can be used for modulation of thermosensitive ion channels without cell damage [71, 72].

The photothermal mechanism described above assumes the energy conversion of photons to phonons of the NPs, which triggers the chemical transformation. An alternative mechanism involves the energy conversion of photons to energetic charge carriers. These carriers can transfer from the NP to adsorbate before their interaction with phonons and following energy dissipation. The efficiency of this process depends on overlapping of electronic states of the NPs and adsorbates as well as lifetime of exited carriers. In case of efficient excitation of charges, their spatial separation and following transfer to the adsorbates should occur. The injection of electrons or holes to adsorbates should be considered separately; nevertheless, it results to the same effect mentioned above.

\subsubsection{Desorption (photons to energetic charge carriers)}

Similar to the desorption caused by light-induced heating and utilised for drug and gene delivery, the electroninduced mechanism demonstrates less efficiency, but higher stability of the adsorbates. For example, pulsed laser radiation can break thiol Au-SH bonds by hot-electron transfer for double-stranded DNA release from the NP surface [15]. We omit here the detailed discussion, as this process is well described in [73], and the corresponding mechanism (desorption induced by electronic transition) is also discussed in previous works $[65,74,75]$.

\subsubsection{Photodissociation and decomposition (photons to energetic charge carriers)}

In general, after electron and hole separation by light and before their collision, these energetic charges can be transferred to the adsorbates, which can result in different effects. Energetic holes of the NPs serve as principal oxidants and are involved in different processes as generation of reactive hydroxyl radicals $(\mathrm{OH} \cdot)$ [76, 77], water splitting $\left(\mathrm{H}_{2} \mathrm{O}+2 \mathrm{~h}^{+} \rightarrow 2 \mathrm{H}^{+}+1 / 2 \mathrm{O}_{2}\right)$, and reactive oxygen species (ROS) $\left(\mathrm{H}_{2} \mathrm{O}+\mathrm{h}^{+} \rightarrow \mathrm{H}^{+}+\cdot \mathrm{OH}\right)$ [78-80], as well as oxidation of citrate molecules and ethylene adsorbed on the NPs [81-83] and many others. In contrast, energetic (hot) electrons participate in hydrogen evolution $\left(2 \mathrm{H}^{+}+2 \mathrm{e}^{-} \rightarrow\right.$ $\mathrm{H}_{2}$ ), generation of superoxide anion $\cdot \mathrm{O}_{2}^{-}$[80], dissociation of oxygen to $\mathrm{O}_{2}^{-}$ions [82], hydrogen dissociation, reduction of nitroaromatic compounds, ammonia decomposition $\left(2 \mathrm{NH}_{3} \rightarrow \mathrm{N}_{2}+3 \mathrm{H}_{2}\right)$ [84], hydrogen production from ammonia borane, and esterification of benzaldehyde with alcohol to produce ethyl benzoate [74, 85-88]. Moreover, for the most complex systems such as Saccharomyces cerevisiae functionalised by indium phosphide NPs, the electron injection from the illuminated NPs to such heterotrophs stimulates cytosolic regeneration of redox cofactor nicotinamide adenine dinucleotide phosphate [89], whereas the complex of Moorella thermoacetica-CdS NPs represent the electronic transfer from the NPs to bacteria for photosynthetic reduction of $\mathrm{CO}_{2}$ to produce acetic acid [90]. However, in case of dielectric NPs, such catalytic activity is limited due to the large band gaps (several eV) of common semiconductors as titanium dioxide $\left(\mathrm{TiO}_{2}\right)$ and very low mobility of their charge carriers. Additionally, it should be noted that, in contrast to the heat-induced dissociation and decomposition, where the NPs and adsorbates are in thermal equilibrium, the charge-induced reaction allows exciting specific vibrational modes of the adsorbates, selectively increases its temperature, and triggers specific reaction.

Finally, resonant interaction between light and the NPs can result in electromagnetic field localisation near the NP surface [91]. In this case, plasmonic NPs demonstrate unprecedented values of field localisation on their surface or in the gaps, whereas dielectric NPs demonstrate the electromagnetic field localisation mostly inside their volume with slight dissipation of energy to their surface $[52,92]$. In any case, the enhancement of electric field $|E|^{2}$ on the surface increases the rate of electron-hole pairs formation in adsorbates that stimulate their chemical transformation [93] via the generation of singlet oxygen ${ }^{1} \mathrm{O}_{2}$ on the surface of metallic and dielectric NPs [80]. Moreover, the energy transfer can be carried out both from the NP to adsorbates, and vice versa [62]. In the latter case, the energy transfer is described by NP surface energy transfer mechanism [94], where an optically excited organic fluorophore acts as a donor, which transfers its energy by dipole-dipole interaction to acceptor (NPs) through a nonradiative path. The efficiency of this process directly depends on overlapping of donor emission spectrum and 
acceptor absorbance. The distance between donor and acceptor [94] and the size of acceptor [95] also play a critical role in the energy transfer efficiency.

\section{Light-responsive plain delivery systems: recent applications in nanomedicine}

\subsection{Plain plasmonic NPs}

As described above, plasmonic NPs (e.g. Au and Ag) have proven to show strong scattering and absorption of light in visible and NIR region owing to their localised surface plasmon resonances (SPRs). The absorbed light is then transformed into thermal energy [96]. Being a good nanoheaters, the $\mathrm{Au}$ and Ag NPs provide a versatile and multifaceted platform for a broad range of biomedical applications such as drug delivery.

\subsubsection{Au NPs}

Among various light-responsive delivery platforms, $\mathrm{Au}$ NPs have attracted tremendous interest as drug carriers due to their particular features; simple synthetic manipulation; enabling precise control over the physicochemical properties of particles; and strong binding affinity to thiols, disulphides, and amines, which allow performing surface coating [97]. At present, various synthetic conditions have been developed for the fabrication of anisotropic Au NPs with different sizes and shapes. Based on dimensions, Au NPs can be divided into three categories: (i) one-dimensional Au NPs (e.g. nanorods, nanowires, nanotubes, etc.); (ii) two-dimensional Au NPs (e.g. stars, pentagons, squares/rectangles, dimpled nanoplates, hexagons, truncated triangles), and (iii) three-dimensional $\mathrm{Au}$ NPs (e.g. nanotadpoles, nanodumbbells, nanopods, nanostars, and nanodendrites). As mentioned above, the SPR band of Au NPs depends on the morphology (e.g. shape, size, core charge, etc.) of Au NPs. A variety of Au NPs with different morphologies and sizes were fabricated to manipulate the visible and NIR spectrum. As an example, recently developed anisotropic popcorn-shaped $\mathrm{Au}$ NPs possessed tunable SPR, high colloidal stability, and high biocompatibility [98].

The effective SPR absorption of Au NPs allows employing these NPs in photodynamic therapy (PDT). Moreover, the modification of Au NPs with PSs allows enhancement of the PDT efficiency in cancer treatment. For instance, in the recent work, generation of singlet oxygen was effectively tuned by manipulating the physical location between Au NPs and PSs [99]. Usually, the synergy of Au NPs and PSs occurs in the following manner: the energy transferred from the Au NPs to the PSs enhances the photodynamic effect with higher stability. After administration of Au NPs in vivo and reaching the tumour cells, ROS are released from the PSs attached to the surfaces of $\mathrm{Au}$ NPs under irradiation. The released ROS demonstrate phototoxic features, whereas nonphototoxic effects are usually observed in the blood circulation without exposure to light. Thus, this synergy of plasmonic NPs with attached PSs demonstrates selectivity of the used therapy.

As mentioned above, the heating abilities of Au NPs make it possible to utilise them also in PTT. It was demonstrated that the heat causes the death of malignant tumours [100]. In general, based on many reports, spherical solid Au NPs with diameter greater than $50 \mathrm{~nm}$ are the most commonly used platform in PTT. Recently, Au NPs were combined with other nanostructures. For example, Chen et al. [101] combined Au NPs with NaYF4, $\mathrm{Yb}^{3+} / \mathrm{Er}^{3+}$ nanomaterials and coated the resulted structure with silica shell. The surfaces of these nanocomposites were then decorated with folic acid through multiple steps to impart the Au NPs properties of selectivity in vivo. The $\mathrm{NaYF} 4, \mathrm{Yb}^{3+} / \mathrm{Er}^{3+}$ nanostructures acted as light converters and emitted green and red light under $980 \mathrm{~nm}$ laser excitation. The additional silica coating improved biocompatibility of the developed systems. This system exhibited significantly enhanced therapeutic efficiency as demonstrated in vitro and in vivo.

The Au NPs can be also employed as effective nanocarriers that are capable of carrying various drugs such as peptides, proteins, plasmid DNAs, small interfering ribonucleic acids, and chemotherapeutic agents [97]. Various surface modification strategies of Au NPs were developed, which allow controlling the penetration and retention of NPs in tumours. For instance, polyethylene glycol (PEG) coating is one of the widely used methods for Au NP modification to prolong the blood circulation time of NPs and enhance their accumulation into tumour cells. Also, the surface of $\mathrm{Au}$ NPs can be modified with antibodies that allow performing targeted delivery of drugs to the area of interest. This enables the combination of the targeted properties of Au NPs with the therapies (e.g. PDT, PTT). In the recent work, Au NPs were loaded with a variety of antitumour agents, including paclitaxel, methotrexate, daunorubicin, gemcitabine, platinum complexes, doxorubicin (DOX), etc. [102]. In this case, loading was performed by simple physical adsorption of antitumour agents on Au NPs or by using alkanethiol linkers. The successful loading of antitumour 
drugs was probed both in vitro on tumour cell cultures and in vivo using mice bearing implanted tumours of various natures and localisations (Lewis lung carcinoma, pancreatic adenocarcinoma, and so forth) [102].

The light sensitivity of Au NPs allows controlling the release process of the drugs. In many works, the light stimuli were used to release drug molecules from Au NPs. For example, Sreejivungsa et al. [103] developed a novel monolayer of Au NPs for the controlled release of a model drug using UV light. Wang and colleagues fabricated multifunctional Au NPs loaded with DOX for the treatment of metastatic breast cancer [104]. Upon laser irradiation, the local temperature of Au NPs was increased substantially and efficiently released DOX from the Au NP surface. In another work, Zhang and colleagues developed a core-shell NPs that consisted of Au nanoshell and 10-hydroxycamptothecin for the treatment of breast cancer [105]. After the introduction of the developed NPs in vivo and their irradiation at power density of $1 \mathrm{~W} \mathrm{~cm}^{-2}$ with the exposure time of $10 \mathrm{~min}$, full tumour remission in a 4T1 breast syngeneic mouse model was observed. It is important that no significant weight loss of mouse and tumour recurrence were indicated. Park and coworkers [106] designed PEGylated multifunctional hollow Au NPs for delivery of DOX against A549 lung cancer. Upon NIR irradiation, the Au NPs efficiently released DOX. Furthermore, these NPs were capable of sensitising A549 cells to X-ray radiation. The effective combination of chemotherapy (DOX), PTT (high temperature), and radiation resulted in the highest cytotoxic effect of the developed systems. In another study, Si phthalocyanine 4 (a drug for the treatment of rabies) was linked to PEGylated Au NP conjugates. After reaching the tumour site and further passive accumulation of NPs, the molecules of phthalocyanine 4 were released from the surface of the NPs under irradiation with light at $670 \mathrm{~nm}$ [107]. Recently, Zhang et al. [108] developed a new Au NP with immunological properties that possessed core-shell structure. These NPs could release heat (PTT) and tumour antigen-based immunotherapeutic agent. The obtained results clearly demonstrated the accumulation of Au NPs in the tumour area, for example, in tumour-bearing mice in $24 \mathrm{~h}$ after administration (Figure 7B) and, therefore, improved antitumour effect from both PTT and immune therapy (Figure 7C).

When speaking about Au NPs, photoporation should be mentioned as a valuable approach to deliver various bioactive molecules in a controlled manner. The mechanism of photoporation is based on the large absorption cross-section of Au NPs and their ability to raise temperature upon irradiation. After incubation of Au NPs with cells, Au NPs absorbed onto cell membranes can permeabilise membranes upon laser irradiation through thermally induced phenomena, local heating, acoustic waves, or formation of water vapour bubbles. Indeed, Xiong et al. [109] used $70 \mathrm{~nm} \mathrm{Au} \mathrm{NPs,} \mathrm{which} \mathrm{were} \mathrm{adsorbed} \mathrm{onto} \mathrm{cell}$ plasma membrane after $30 \mathrm{~min}$. Afterwards, compound of interest was added, and cells were irradiated in a controlled manner with laser in pulsed regime. The formation of vapour bubbles induced nanopores in cell plasma membrane and allowed compound of interest to diffuse inside cells. This approach enables selective delivery of bioactive molecules in a spatially resolved manner using $\mathrm{Au}$ NPs [109].

Overall, $\mathrm{Au}$ NPs are the most widely studied drug carriers because of low inherent toxicity, high surface area, and tunable stability. However, some issues still need to be addressed such as engineering of Au NP's surface in order to improve Au NP's bioavailability and immunogenicity. Moreover, Au NPs are not able to protect cargo from degradation in biological fluids, as bioactive compounds are usually attached to the NP's surface, and to keep these molecules intact, additional organic coatings are required.

\subsubsection{Silver NPs}

Silver NPs are also a widely studied plasmonic material that can be used in biomedical applications. An advantage of Ag NPs is simple and low-cost chemical synthesis. Silver NPs can be obtained in various shapes, such as spherical, rod, octagonal, triangle, flower-like, etc. As a drawback, Ag NPs have low biocompatibility and, therefore, cytotoxicity to healthy cells. The smaller-sized Ag NPs cause enhanced cytotoxic effects compared to the larger counterparts due to the increased surface reactivity of NPs. The shape of Ag NPs also contributes to overall toxicity. As the size and shape of Ag NPs are directly related to the SPR, it is important to optimise the Ag NP morphology in order to achieve the sufficient biocompatibility and effective light sensitivity. As for Au NPs, the surface of Ag NPs can be coated with biopolymers, which can reduce their cytotoxicity. By varying the size and shape, it is possible to tune the plasmon peak of Ag NPs in the range of 393-738 nm and $500-1000 \mathrm{~nm}$, which leads to strong visible and NIR scattering and absorption enabling employment Ag NPs in PTT and PDT [110].

Similar to Au NPs, Ag NPs are also good nanoheaters, and this can be effectively employed in PTT. In the recent work, Ag-based NPs were developed with the improved ability to absorb NIR irradiation. The developed systems could perform PTT therapy against A549 cells at a low irradiation power density $\left(0.20 \mathrm{~W} \mathrm{~cm}^{-2}\right)$ without any damage 


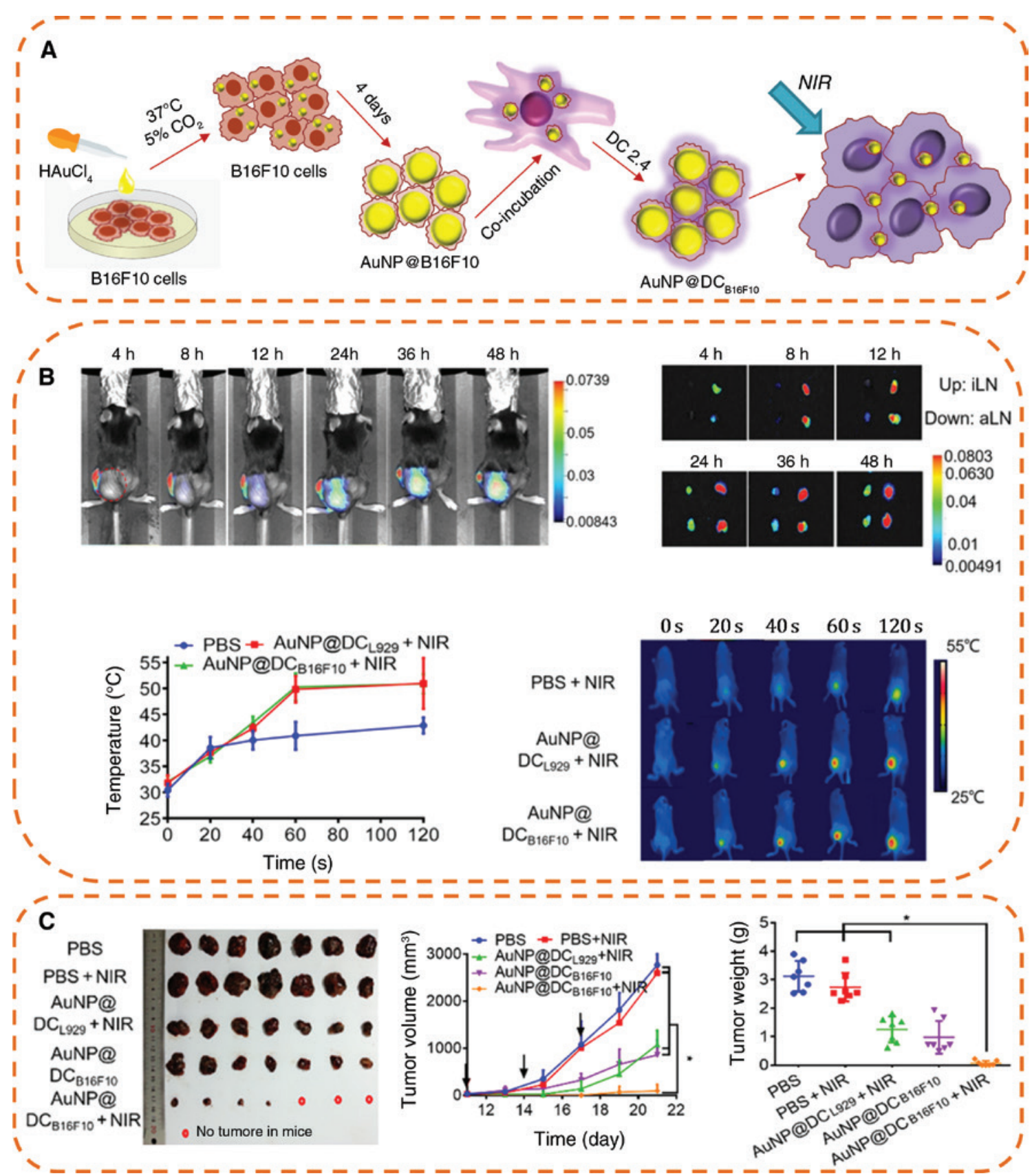

Figure 7: In vivo characterisation and antitumour effect of immunological Au NPs combined PTT and tumour antigen-based immunotherapy. (A) Schematic diagram of PTT using Au NPs. (B) In vivo distribution of Au NPs labeled with fluorescent marker in tumour-bearing mice at different time points. IR thermal images with corresponding temperature profiles. (C) The photographs of tumours from the sacrificed mice at the 21st day. Tumour growth curves after different treatments with and without laser irradiation of $2.0 \mathrm{~W} \mathrm{~cm}^{-2}$. Tumour weight of the sacrificed mice at the 21st day. Adapted with permission from [108]. Copyright 2019 American Chemical Society.

to the normal healthy cells and the surrounding tissues [111]. Another study reported that DOX-loaded Ag-based NPs demonstrated excellent chemophotothermal therapeutic efficiency and possessed NIR-laser-controlled drug-releasing functions [112].

Several researchers developed Ag NP-based PSs for PDT [113]. In general, to fabricate Ag NP-based PSs, bovine serum albumin (BSA) is used [113]. The functional groups of BSA (e.g. amino, imidazolyl, and thyol) interact with Ag ions forming stable Ag-BSA complexes. For example, Cui and colleagues synthesised Ag nanodots using BSA via biomineralisation method [114]. The diameter of Ag nanodots was $5.80 \pm 0.5 \mathrm{~nm}$, which corresponded to the most optimal photothermal properties. Later, Vankayala et al. 
[115] showed that singlet oxygen can be formed through direct sensitisation using Ag NPs without presence of any organic PSs under light irradiation.

Some studies reported that the fast biodegradation of $\mathrm{Ag}$ NPs on the cellular level and within a whole organism can significantly limit the application of Ag NPs for drug delivery applications. Motivated by the high plasmonic potential of Ag NPs, several strategies were suggested to preserve $\mathrm{Ag}$ nanostructure from the fast degradation [116]. These strategies mostly focus on surface modification of Ag NPs with an extra protecting layer of inorganic or organic coating, such as $\mathrm{Si}$ [117], graphene oxide (GO) [118], self-assembled monolayers of organic thiols [119], etc. Another approach to protect the unstable Ag NPs involves $\mathrm{Au}$ coating. As discussed above, the $\mathrm{Au}$ NPs possess own plasmonic resonances in combination with slow biodegradation in the biological environment. The result of Ag modification with Au nanostructures can lead to the enhanced plasmonic properties. Up to now, there are only few studies employing $\mathrm{Ag}-\mathrm{Au}$ NPs as light-sensitive drug delivery carriers $[120,121]$. Nevertheless, the PTT potential and fate in the biological environment of Ag-based NPs are not fully explored. Recently, Espinosa et al. [116] fabricated anisotropic hybrids composed of Ag NP cores coated with Au shell, which possessed efficient NIR absorption. For this synthesis, Au nanostars and Ag

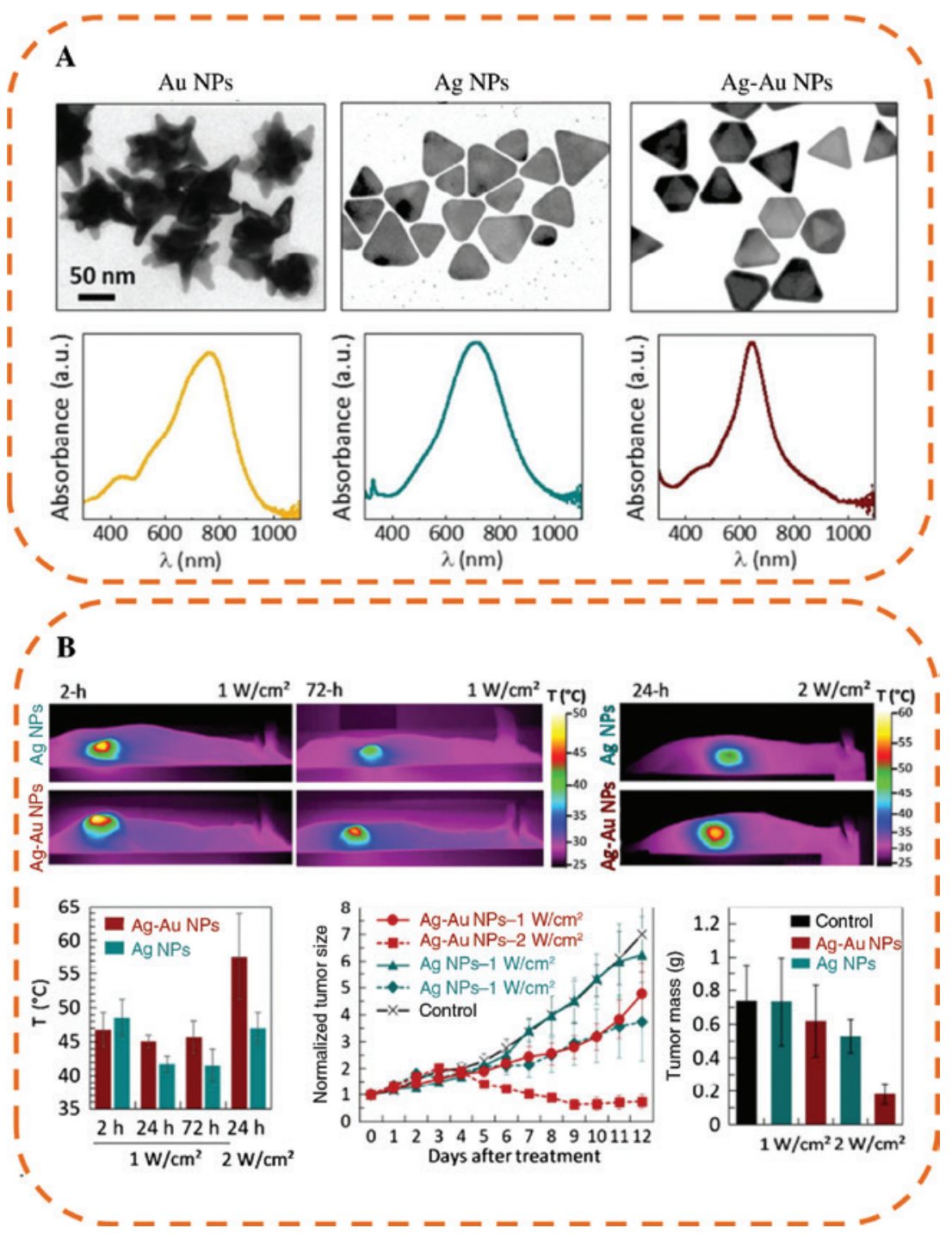

Figure 8: Structure and photothermal efficiency of $\mathrm{Ag}, \mathrm{Au}$, and $\mathrm{Ag}-\mathrm{Au}$ NPs.

(A) Transmission electron microscopy (TEM) images and UV-visible/NIR spectra of Au NPs, Ag NPs, and Ag-Au NPs. (B) Infrared (IR) thermal images of mice injected with Ag NPs and Ag-Au NPs exposed under NIR-laser irradiation $\left(680 \mathrm{~nm}, 1 \mathrm{~min}, 1 \mathrm{~W} \mathrm{~cm}{ }^{-2}\right)$ with corresponding analysis of average temperature after $1 \mathrm{~min}$ of $680 \mathrm{~nm}$ NIR-laser exposure and effect on tumours. Reprinted with permission from [116]. Copyright 2018 American Chemical Society. 
nanoplates were used (Figure 8A). The evaluation of the photothermal efficiency and intracellular biodegradation of the developed Ag-Au-based NPs was tested on the tissue-mimetic model in vivo. According to the obtained results, almost total tumour regression was observed on the example of mice model. Moreover, in the comparative study, it was demonstrated that the cellular heating was much more efficient in case of Ag-Au NPs compared to individual Ag NPs. This indicated the high potential of $\mathrm{Ag}-\mathrm{Au}$ NPs in PTT due to improved heating properties of the developed carriers (Figure 8B). Thus, the surface modification of Ag NPs by Au coating provides new functional properties for developing efficient drug delivery platform.

\subsection{Plain dielectric NPs}

Despite wide applicability of plasmonic NPs as light-sensitive drug delivery carriers, Au and Ag NPs have finite conductivities at optical frequencies. This can lead to the inherent dissipation of the electromagnetic energy [122]. Moreover, fluorescent compounds placed near plasmonic NPs experience quenching effects. To overcome this, the additional spacer on plasmonic NP's surface is needed to increase the distance between the fluorescent molecule and surface of NP [123]. Alternatively, the high refractive index dielectric NPs are under intensive investigation. Owing to their better resonant properties, dielectric NPs such as $\mathrm{Si}, \mathrm{Ge}$, and $\mathrm{Fe}_{2} \mathrm{O}_{3}$ can be more efficient heaters compared to plasmonic counterparts [26]. Therefore, resonant dielectric NPs have a great potential to be used as drug delivery carriers, enabling remote controlled activation of drugs under optical irradiation.

\subsubsection{Si NPs}

One of the most abundant light-sensitive materials that can be also used in nanomedicine is $\mathrm{Si}$. The most common approach to fabricate crystalline Si NPs is laser ablation, resulting in an effective nanoheater with excellent resonant properties [26, 124]. Alternatively, electrochemical etching approach for the Si NP synthesis leads to the formation of porous Si NPs, which can be effectively loaded with drugs. Xia et al. [125] demonstrated an increased delivery of the commercially available drug DOX into cancer cells using light-sensitive porous Si NPs. These carriers were loaded with a transducer (indocyanine green), which can effectively convert light into heat. NIR irradiation of the internalised carriers resulted in the increased release of DOX compared to the $\mathrm{pH}$-induced release in the acidic microenvironment of lysosomes [125]. Another study reported on the excellent heat generation abilities of porous Si NPs under NIR irradiation itself enabling PTT. In vivo animal tests on the Balb/c mice revealed murine colon carcinoma (CT-26) tumours were completely resorbed within 5 days after porous Si NP treatment in combination with NIR laser [126]. Apart from PTT, porous Si NPs can provide efficient PDT, which involves the conversion of ground-state molecular oxygen $\left({ }^{3} \mathrm{O}_{2}\right)$ to singlet oxygen $\left({ }^{1} \mathrm{O}_{2}\right)$ by energy transfer. As mentioned, the ${ }^{1} \mathrm{O}_{2}$ is highly reactive and can cause the lethal damage of target cells [127]. When excited with visible light, the quantum-confined domains in porous $\mathrm{Si}$ NPs generate ${ }^{1} \mathrm{O}_{2}$, and due to extremely long exciton lifetime, ${ }^{1} \mathrm{O}_{2}$ generation is efficient [128]. Indeed, Xiao et al. [129] demonstrated an improved PDT from porous Si NPs prepared by electrochemically etched Si wafers in cancer cells (HeLa and NIH-3T3). According to the obtained data, $45 \%$ of cells were dead after PDT treatment compared to the less than $10 \%$ cytotoxicity in control experiments [129]. Si NPs can be also used as carriers for the PSs that are designed to generate ROS. Secret et al. [130] covalently linked porphyrin to the Si NPs in order to demonstrate imaging and PDT in vitro under two-photon excitation (TPE) conditions. The authors demonstrated that PDT occurred under TPE conditions, where Si NPs transferred light energy to porphyrin, resulting in MCF-7 breast cancer cell death [130]. Other PSs can be used to modify Si NPs for PDT. Knežević et al. [131] employed ruthenium (Ru) (II) complexes that can adsorb visible and UV to functionalise Si NPs. The surface of Si NPs was additionally linked with targeting moieties to enable selective targeting to tumour tissues. The developed systems demonstrated increased cytotoxicity under exposure to blue and NIR light [131]. The fluorescent small-sized (4 nm) Si quantum dots (QDs) were also applied as PSs (chlorin e6) carriers for PDT. Additionally Si QDs were modified with $\mathrm{MnO}_{2}$, which is known to produce oxygen in the reaction with the endogenous excess metabolites $\left(\mathrm{H}_{2} \mathrm{O}_{2}\right)$ in acidic tumour microenvironment. The developed Si-based carriers enabled enhanced loading capacity with PSs and dualimaging properties [fluorescence and magnetic resonance tomography, magnetic resonance imaging (MRI)], which resulted in significant inhibition of HeLa-bearing tumour in vivo [132].

\subsubsection{Titanium dioxide NPs}

Another light-responsive material that can be used as drug delivery carriers is $\mathrm{TiO}_{2}$. An important feature of $\mathrm{TiO}_{2} \mathrm{NPs}$ is its photocatalytic properties, which can be activated under visible or UV light. This results in generation of 
cytotoxic ROS such as hydrogen peroxide and peroxy radicals, which can be harmful for targeted cells [133]. Unlike $\mathrm{Si} \mathrm{NPs}, \mathrm{TiO}_{2}$ NPs are mostly synthesised using chemical methods, such as solvothermal method, sol-gel methods, and so forth [134]. This allows precise controlling size and shape of $\mathrm{TiO}_{2} \mathrm{NPs}$, which is essential in biomedical applications. Liu et al. [135] probed photocatalytic activity of $\mathrm{TiO}_{2} \mathrm{NPs}$, which were attached onto the surface of $\mathrm{TiO}_{2}$ nanotubes in Streptococcus mutans and Porphyromonas gingivalis bacteria. The developed photosensitive material demonstrated improved antimicrobial properties under UV light due to decreased size of $\mathrm{TiO}_{2} \mathrm{NPs}$ and therefore increased surface area and greater amount of released toxic ROS [135]. In another study, Yadav et al. [136] realised the combined cancer therapy using coral-shaped $\mathrm{TiO}_{2}$ NPs loaded with anticancer drug DOX. The increased surface area of $\mathrm{TiO}_{2} \mathrm{NPs}$ resulted in increased payload of DOX onto the NPs. The developed delivery systems provided synergetic cytotoxic effect in cancer MCF7 cells from the released DOX (under biological stimuli inside cells) and generation of ROS from the $\mathrm{TiO}_{2} \mathrm{NPs}$ (under UV-light irradiation) [136]. From clinical perspective, the photoactivation of $\mathrm{TiO}_{2}$ NPs with UV light has several limitations, such as low tissue penetration depth [137], and UV-mediated ROS production lasts for a short time and is not enough to provide a sustained and prolonged tumour destruction [138]. To address it, the drug delivery systems based on $\mathrm{TiO}_{2} \mathrm{NPs}$, which generate ROS under UV-light irradiation, can be loaded with visible light-responsive PSs. Indeed, $\mathrm{TiO}_{2} \mathrm{NPs}$ were loaded with $\mathrm{Ru}$ complexes, which are already in clinical trials as anticancer agents [139]. Ruthenium complexes are able to generate ${ }^{1} \mathrm{O}_{2}$ under visible light irradiation. The developed systems showed sensitivity to UV and visible (green) light as demonstrated in melanoma cells [140]. Another way to overcome the limitation of UV light is represented by the X-ray-responsive mesoporous $\mathrm{TiO}_{2}$ NPs that can be also employed for PDT. In the recent study, Guo et al. [141] designed an effective drug delivery platform that possessed combined cancer therapy (PDT and chemotherapy) based on mesoporous $\mathrm{TiO}_{2}$ NPs. These carriers were loaded with anticancer drug DOX. Additionally, surface of mesoporous $\mathrm{TiO}_{2} \mathrm{NPs}$ was modified with targeting moieties [hyaluronic acid (HA) and cyclic pentapeptide]. The developed systems demonstrated an increased selectivity to CD44-overexpressing tumour cells and improved cytotoxicity [141]. Another approach to avoid irradiation with UV light of biological objects and induce generation of ROS is to combine $\mathrm{TiO}_{2}$ NPs with upconverting NPs (UCNPs) such as rare earthbased NPs [142-144]. As mentioned above, NIR light is able to penetrate deeper into cells and tissues and has minimal photodamage [145]. One of the main features of the UCNPs is their ability to absorb light energy in NIR region and convert it into UV light due to the unique ladder-like structure of energy levels of lanthanide ions [144]. Yin et al. [146] developed NaGdF4, Yb, Tm NPs as a core, with the $\mathrm{TiO}_{2}$ as a shell for NIR-mediated combined anticancer therapy. The chemotherapy was achieved by loading of the developed carriers with DOX, and PDT was provided by $\mathrm{TiO}_{2}$ shell, which expressed ROS under irradiation of whole system with NIR light, which was subsequently converted into UV light. As a result, the developed systems demonstrated an increased cancer cell (MDA-MB231) death [146]. To shift the activation of $\mathrm{TiO}_{2} \mathrm{NPs}$ into the visible light spectrum, $\mathrm{TiO}_{2}$ NPs can be grafted with some transition metal oxides such as iron oxide NPs (IONPs). Therefore, the nanocomposites $\left(\mathrm{TiO}_{2}-\mathrm{IONPs}\right)$ can be applied as visible light-sensitive PSs for PDT. Zhang et al. [147] developed $\mathrm{TiO}_{2}-\mathrm{Fe}_{3} \mathrm{O}_{4}$ complexes, which generated ROS under visible light. Additionally, these complexes were loaded with $\mathrm{Fe}^{2+}$-dependent drug artemisinin, and the surface was modified with HA to induce HA-mediated endocytosis. According to the obtained results, the developed systems induced an improved tumour inhibition in vitro and in vivo (Figure 9B) [147].

\subsubsection{Iron oxide NPs}

Magnetic IONPs are widely used particles (some of them are already clinically approved) in biology and medicine as MRI contrast agents, in magnetic hyperthermia applications, and others [149-151]. Despite the magnetic properties of IONCs, recent studies demonstrated that highly crystalline IONPs possess also thermal response on NIR light [152], which can be effectively used in PTT. The mechanism of photothermal energy conversion has not been fully understood. This phenomenon can be explained by high density of defects in the electronic structure of $\mathrm{Fe}_{3} \mathrm{O}_{4} \mathrm{NPs}$. These defects can be attributed to the mixed valences of neighbouring cation ions $\left(\mathrm{Fe}^{2+}\right.$ and $\left.\mathrm{Fe}^{3+}\right)$ of the inverse spinel-structured $\mathrm{Fe}_{3} \mathrm{O}_{4} \mathrm{NPs}$ [153]. Therefore, the narrowing of the band gap takes place with the consequence formation of oxygen vacancies. The heating then subsequently occurs under NIR light, when optically excited electrons exchange transitions from the different defects, which is related to the different band gap energies [154]. The highly crystalline IONPs are usually synthesised by thermal decomposition method with further coating of NPs with polymers to make them water-soluble, which is essential for biomedical applications. Chen et al. [152] reported on the concentration-dependent 


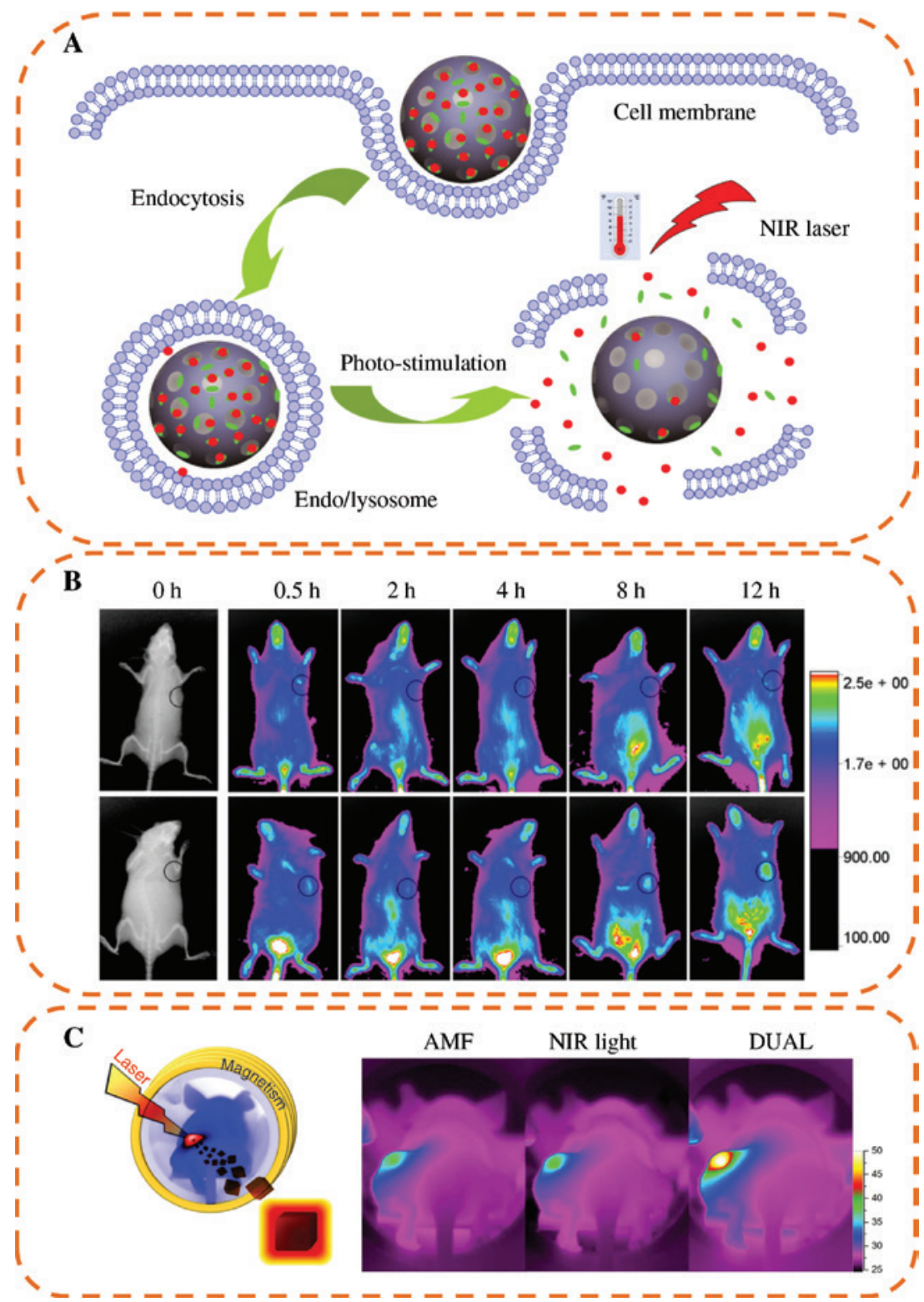

Figure 9: Example of light-treatment with inorganic NPs.

(A) Schematic diagram of employment of silica NPs as light-sensitive drug carriers. (B) In vivo NIR imaging of tumour-bearing mice intravenous injected with free NIR-dye (IR783) solution (upper row) and IR783-loaded $\mathrm{HA}-\mathrm{TiO}_{2}-$ IONPs (lower row) at different times after injection. (C) Thermal images obtained with the IR camera in mouse, after intratumoural injection IONPs, in the left-hand tumour, and after 10-min application of AMF, NIR-laser irradiation, or DUAL (both effects). Reprinted with permission from [125, 147, 148]. Copyright 2015 John Wiley and Sons; Copyright 2017 Impact Journals; Copyright 2016 American Chemical Society.

temperature increase up to $56^{\circ} \mathrm{C}[0.5 \mathrm{mg}(\mathrm{Fe}) / \mathrm{ml}]$ when $\mathrm{Fe}_{3} \mathrm{O}_{4}$ NPs were irradiated with light. After intravenous administration into SUM-159 tumour-bearing mice, IONPs could effectively accumulate within the tumour (5.3\% of injection dose) due to enhanced permeability and retention effect. After NIR irradiation of the tumour zone, complete tumour regression was observed [152]. In another study, Oh et al. [155] showed combined PTT and chemotherapy after administration of highly crystalline $\mathrm{Fe}_{3} \mathrm{O}_{4} \mathrm{NPs}$ loaded with DOX. The enhanced cytotoxicity of human breast cancer cells (MDA-MB-231) was induced by synergetic effect from heat under NIR-laser irradiation and released DOX [155]. Shen et al. [156] developed temperature-responsive drug carriers based on IONPs. In this work, $\mathrm{Fe}_{3} \mathrm{O}_{4}$ NPs were coated with temperature-sensitive polymer, which was postloaded with DOX. The NIR irradiation of $\mathrm{Fe}_{3} \mathrm{O}_{4}$ induced increase in the temperature, which resulted in the polymer shrink with the subsequent release of DOX. These NIR-light-responsive drug delivery systems were tested in vitro and in vivo. The revealed data 
demonstrated tumour inhibition rate of up to $91.5 \%$ [156]. Apart from MRI, the additional functionalisation of IONPs with carbon can provide fluorescence properties enabling dual-modal imaging. Wang et al. [157] developed multifunctional carriers that comprised $\mathrm{Fe}_{3} \mathrm{O}_{4}$ as a core material and carbon as a shell material. These carriers exhibited NIR, upconverted, and wavelength-tunable fluorescence properties. The multimodal imaging and PTT were probed on mice bearing $\mathrm{C} 6$ glioblastoma and resulted in improved tumour therapy [157]. In another study, Wang et al. [158] combined magnetic and upconversion properties in one particulate system for PDT. Optically and magnetically responsive liquid marbles based on $\mathrm{NaYF}_{4}$ nanocrystals and doped with $\mathrm{Yb}^{3+} / \mathrm{Er}^{3+} / \mathrm{Gd}^{3+}$ were loaded with protoporphyrin IX to produce ROS. In the developed system, UCNP outer layer acted as a light transducer to introduce photons with higher energy that are necessary to activate PSs [158]. As mentioned above, highly crystalline IONPs are capable of being heated under alternating magnetic field and NIR irradiation. This feature of IONPs was examined by Espinosa et al. [148], where iron oxide nanocubes were injected into the tumour of 8-week-old female immunodeficient athymic nude NMRI mice. Laboratory animals were treated simultaneously with alternating magnetic field and NIR-laser irradiation. The revealed data showed the twofold to fivefold amplification of heat in the tumour area compared with magnetic stimulation alone. These dual-mode treatment resulted in complete tumour regression (Figure 9C) [148].

\subsubsection{Lanthanide-doped UCNPs}

Recently, UCNPs doped with lanthanide ions have emerged a great potential as drug delivery carriers due to possibility to control carrier fate inside cells and laboratory animals with optical microscopy (deep optical bioimaging). Indeed, UCNPs are able to absorb light with lower energies (NIR) and convert in high-energy radiation (UV/ visible). Detailed explanation of this phenomenon can be found in the studies of Escudero et al. [144] and Bagheri et al. [159]. Therefore, remarkable advantages that include enhanced biological tissue penetration depths, less photodamage, and good biocompatibility make UCNPs preferable as light-responsive delivery carriers. Lanthanide-doped UCNPs of different sizes and shapes can be synthesised with wet chemical methods. These methods are generally based on precipitation reactions. In order to obtain monodisperse uniform NPs, the controlled release of cations and/or anions, as well as appropriate reaction kinetics, is required $[142,143]$.
Apart from bioimaging, UCNPs can be used as a source to induce drug release through photodegradation of PSs that should be incorporated into drug carrier. Indeed, Yao et al. [160] demonstrated light-induced release of anticancer drug from carriers based on UCNPs incorporated into AZO amphiphilic molecules, which are sensitive to NIR to UV/vis upconversion luminescence. They can transform the trans-isomer into the cis-isomer under UV light and conversely the cis-isomer into the trans-isomer under visible light. Authors probed release of DOX using NIR light $(980 \mathrm{~nm})$ on the model of MCF-7/ADR tumour borne by nude mice [160]. In another study, photocleavable moieties were conjugated to the surface of UCNPs to release chemotherapeutic upon light irradiation. For this, UCNPs were coated with $o$-phosphorylethanolamine ligands and coupled to an NB derivative of 5-fluorouracil. Upconverting NPs absorbed NIR light and emitted blue photoluminescence that was in resonance with the absorption band of NB derivative of 5-fluorouracil. This resulted in photocleavage with subsequent release of drug [161]. Another photocleavable delivery system was developed by Xing et al. [162]. Photoresponsive copolymer modified with folic acid was conjugated with UCNPs $\left(\mathrm{NaYF}, \mathrm{Yb}^{3+}, \mathrm{Tm}^{3+}\right)$. The developed carriers were then exposed to NIR light with subsequent release of DOX in a sustained manner [162]. Jalani et al. [163] developed NIR-sensitive delivery systems based on UCNPs $\left(\mathrm{LiYF} 4, \mathrm{Yb}^{3+}, \mathrm{Tm}^{3+}\right.$ ) coated with chitosan hydrogel cross-linked with a photocleavable cross-linker. Under $980 \mathrm{~nm}$ excitation, UV emission cleaved cross-linked and released loaded fluorescent BSA. The efficiency of the remote drug release was tested in vitro [163]. Upconverting NPs can be also used for multiple-drug loading. In the recent study, $\mathrm{NaYbF}_{4}$,Er UCNPs were used as core element for loading of several compounds: (i) antitumour drug DOX and (ii) tocopheryl polyethyleneglycol 1000 succinate to overcome multidrug resistance. Both compounds were liberated from the developed carriers in a prolonged manner in physiological conditions. Moreover, upconversion abilities of carriers and strong X-ray attenuation enabled dualimaging modalities (luminescence and X-ray computed tomography), which made the developed carriers good candidates for imaging-guided cancer agents [164].

\section{Light-responsive composite delivery systems: recent applications in nanomedicine}

Recent developments in material science enable fabrication of light-sensitive drug delivery systems with complex 
architecture taking advantages from individual delivery system. The main goal of composite systems is to design materials that possess novel functional properties such as enhanced drug loading, prolonged circulation times in vivo, multimodal imaging, and others $[22,165]$. In this section, we discuss the recent advances in the development and application of light-sensitive drug delivery systems based on polymers and on biological systems.

\subsection{Composite delivery platforms based on organic NPs}

Organic-based NPs are one of most attractive delivery systems, which can be easily modified with light-sensitive moieties. Light sensitivity of organic NP-based delivery systems is usually achieved through incorporation of specific chemical moieties or nanostructured materials into the polymer structure. There are several organicbased materials, including lipids and polymers, which are usually used for NP fabrication [166]. The advantages of these materials are determined by their reduced toxicity, easy synthesis, and suitability for the drug encapsulation [167]. In this part, we review the most extensively used light-sensitive composite carriers based on (4.1.1) amphiphilic macromolecules, (4.1.2) liposomes, and (4.1.3) polymeric particles.

\subsubsection{Amphiphilic macromolecules (micelles)}

Hydrophobic bioactive compounds can be encapsulated into the cavity of amphiphilic macromolecules, so-called micelles, due to the hydrophilic heads and hydrophobic tails. This leads to the formation of vesicle-like structure with hydrophobic cores [168], and on-demand delivery of

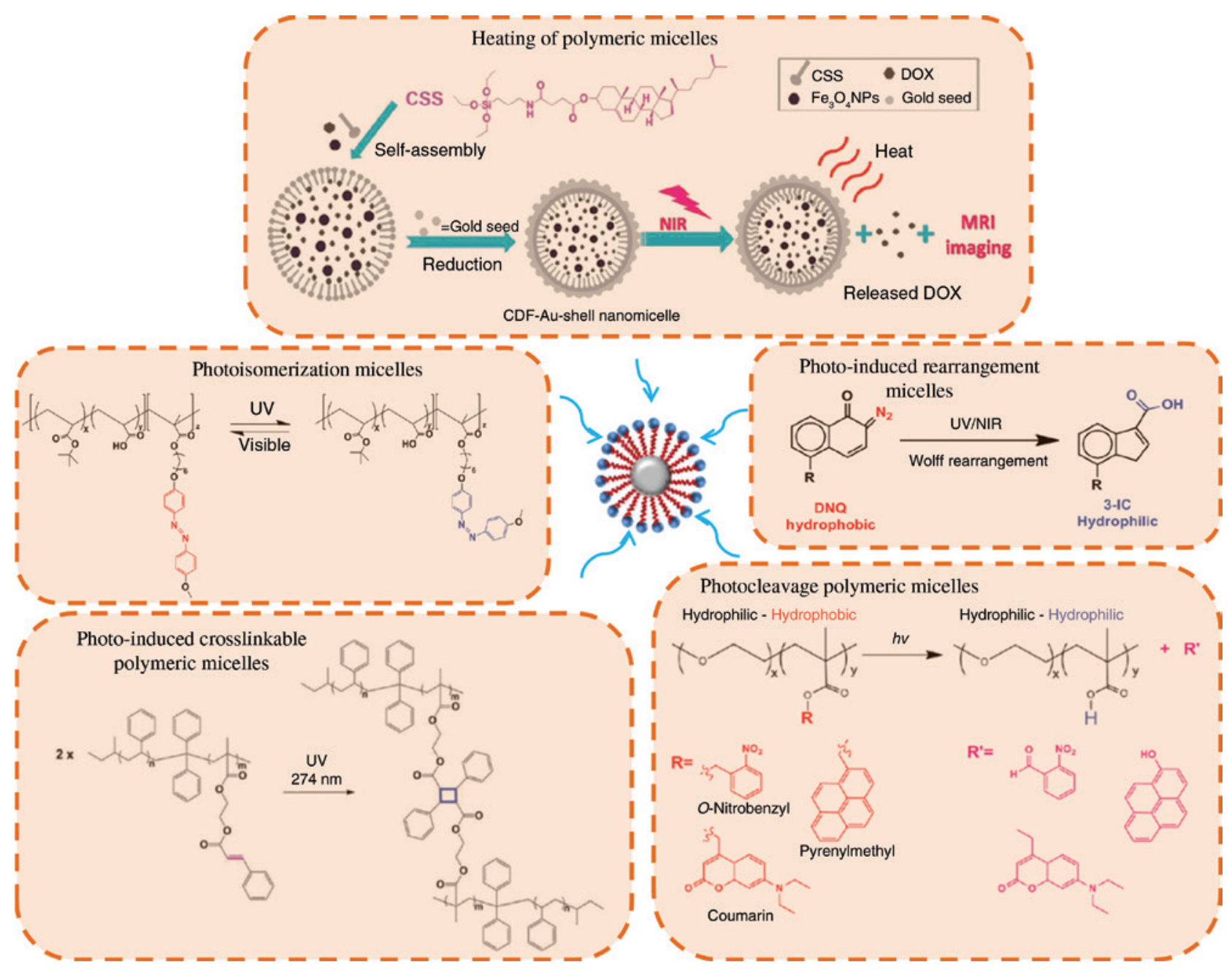

Figure 10: Schematic illustration of different release mechanisms of photo-responsive micelles. Reprinted with permission from [168]. Copyright 2014 Royal Society of Chemistry. 
drugs occurs by disrupting the hydrophilic-hydrophobic balance of the carriers. Micelles are usually synthesised out of biocompatible materials [169] with the incorporated PSs in its structure, which convert photoirradiation into a chemical signal. This conversion occurs through the different types of photoreactions such as isomerisation, rearrangement, cleavage, dimerisation, and heating, which are comprehensively described by Huang et al. [168] (Figure 10).

\subsubsection{Isomerisation}

This process is accomplished by the structural changes in the micellar structure upon light irradiation. The typical photoisomerisation molecules, which are used to design polymeric micelles, include AZO, SP, and dithienylethene [157]. Molla et al. [170] fabricated AZO-containing photoisomerisation micelles using b-PEG-azoB-PLA. The developed systems allowed encapsulation of hydrophobic and hydrophilic molecules and their further release upon UV light [170]. Yuan et al. [171] also designed novel amphiphilic AZO-containing micelles for an effective light-induced destruction of micelles upon UV light (360 nm).

\subsubsection{Rearrangement}

Upon light irradiation, the hydrophobic part of amphiphilic polymers converts into hydrophilic ones, which results in disassembly of polymeric micelles. An example of such molecule involved in this process is hydrophobic DNQ molecule, which can be changed into a hydrophilic 3-indenecarboxylic acid upon UV-light irradiation (Figure 10) [168]. Li et al. [172] developed novel DNQ-containing multifunctional light-responsive micelles, where DNQ groups can undergo Wolff rearrangement under UV/ NIR light irradiation.

\subsubsection{Cleavage}

This mechanism is based on the break of photocleavage groups located in the side chain of the micelles. Photocleavage reaction usually occurs using photochromic NB and coumarin-incorporated copolymers, which are employed in fabrication of light-responsive micelles. As an example, Ji et al. [173] showed photoresponsive poly(ethylene glycol)-block-poly(o-nitrobenzyl-L-glutamate) diblock copolymers. These copolymers can self-assemble into spherical micelles in aqueous solution. The obtained micelles demonstrated photoinduced transition of their morphology from spheres to cylinders under UV light. This phenomenon arises from the disruption of amphiphilicity induced by the cleavage of NB groups on the side chains [173].

\subsubsection{Dimerisation}

This mechanism of conformational changes in micellar structure is based on temperature-dependent photoinduced chemical bonds, which turn into the swollen state above the lower critical solution temperature (LCST). This results in the release of the pre-encapsulated drug. In the recent study, dual-responsive polymeric micelles were designed using photodimerisation process, which were capable of tuning the LCST of the polymer by attaching photosensitive moieties [174].

\subsubsection{Heating}

The heating process is associated with the conversion of light energy into heat. For this, the polymeric micelles are usually modified with inorganic particles ( $\mathrm{Au}, \mathrm{Ag}$, and other), which possess NIR absorption. Ma et al. [175] reported on $\mathrm{Au}$ nanoshell nanomicelles with light-triggered release mechanism for PTT. Authors demonstrated an efficient NIR-induced stepwise release behaviour of DOX in vitro.

Therefore, by the application of each previously mentioned mechanism, one can control the stabilisation of the micellar structure, formation/disruption of micelles, or cross-linking.

\subsubsection{Liposomes}

The great step forward in preclinical and clinical studies was made in employment of liposomes as drug delivery carriers. The synthesis of liposomes is rather straightforward and can be achieved in size-controlled manner [176]. Liposomes usually comprised of naturally derived phospholipids, which can mimic the biological membranes [172]. Unlike micelles, hydrophilic and hydrophobic molecules can be loaded into the structure of liposomes because of its lipid bilayer structure. Regarding the synthesis and loading of liposomes with bioactive compounds, we refer to the following review articles [176-178].

Liposomes can be further engineered with functional moieties to be light-sensitive. The most common way to do this is to include PSs that transfer the light energy to molecular oxygen with further formation of ROS. Generally, PSs are hydrophobic; therefore, encapsulation of these compounds in the liposomes increases their solubility, as well as delivery to the targets [179]. The mechanisms of the light-triggered release of cargo from the liposomes can be different and are similar to the micelles. They mostly include photoisomerisation, photocleavage of lipids upon exposure to light-mediated formation of ROS, 
and photopolymerisation of lipids containing double or triple bonds. Another way to stimulate release of bioactive compounds from liposomes under light is incorporation of plasmonic nanostructures. Au NPs can be embedded into the liposomal structure either via thiol bonding or via electrostatic interactions, when mixing formed liposomes with Au NPs [180]. Under laser irradiation, because of the large temperature gradients, the surrounding medium produces bubbles, which induce rupture of liposomal structure with the consequent release of cargo into the surroundings [181]. However, Palankar et al. [182] demonstrated that apart from rupture transient pores in liposomal membrane can occur upon localised laser irradiation of $\mathrm{Au}$ NPs embedded into the structure of liposomes. This transient opening of liposomal membrane was monitored by ion current. Another mechanism of cargo release from liposomes modified with Au NPs is the thermic effects, which lead to the disintegration or melting of liposomal structure. For instance, Luo et al. [183] showed controlled release upon NIR irradiation from chitosan-coated oleic acid liposomes with outer layer of Au NPs. The mechanism of the release in this case was activation of the gel to liquid crystalline phase transition of the liposomes [183]. In another study, Lajunen et al. [184] demonstrated lighttriggered release of fluorescence probes from liposomes that contained DPPC (1,2-dipalmitoyl-sn-glycero-3-phosphocholine) and DSPC (1,2-distearoyl-sn-glycero-3-phosphocholine) and modified either with Au nanorods or $\mathrm{Au}$ nanostars. The release of cargo was temperature dependent, proving that with the increase in temperature disintegration/melting rate of liposomes also increases [184]. Light-responsive liposomes can be additionally designed for addressable delivery of bioactive compounds. For this, apart from functionalisation with $\mathrm{Au}$ NPs (for light sensitivity), surface of liposomes can be modified with targeting molecules (antibodies, peptides). Indeed, Li et al. [185] conjugated human epidermal growth factor receptor 2 antibody to the surface of liposomes. As a result, authors observed approximately twofold increase in accumulation of carriers in vivo near the tumour region. The targeted delivery did not cause systemic toxicity after injection [185]. In another study, peptides were applied to improve cellular uptake of light-sensitive liposomes. For this, SPACE (skin-penetrating-and-cell-entering) peptides were conjugated onto the surface of liposomes, which were in turn modified with Au NPs. Fluorescence molecules and nucleic acids were delivered with the developed carrier systems, and the release was triggered upon NIR irradiation. The authors claimed the mechanism that induced the intracellular release was the vapour bubble formation, which led to the mechanical disruption of liposomes [186].
In the recent in vivo study, Li et al. [187] combined PDT and chemotherapy in one light-responsive liposomal carrier. For this, PSs (ICG-ODA) as well as DOX were incorporated into the structure of liposomes. The combined therapy led to the significant inhibition of tumour growth compared to the PDT or chemotherapy alone [187].

\subsubsection{Polymers}

Light-responsive polymeric delivery systems are under extensive investigation in the past decade. The basic principle of polymer carriers' synthesis is comprehensively described in the recent review article [22] and based on so-called layer-by-layer technique, which consists of deposition of oppositely charged polymers onto the sacrificial template [188]. After the dissolution of template, hollow polymer capsules can be produced, which in turn can be loaded with various bioactive compounds, such as drugs [189], nucleic acids [190], macromolecules [9], fluorescent moieties [191], and other. Generally, to impart polymer capsules properties of light sensitivity, their shell should be modified with plasmonic/dielectric NPs. These NPs can be simply attached onto the polymeric shell via electrostatic interactions. The main mechanism of intracellular cargo release from the polymer capsules upon laser irradiation is the temperature increase, which leads to the change of the permeability of the polymer shell and thus the triggered release of cargo [9, 192, 193]. The detailed description of thermodynamics and temperature rise inside polymer capsules modified with plasmonic NPs is provided in the recent works [194, 195]. In the pioneer works, aggregated $\mathrm{Au}$ NPs were employed as heat agents embedded into the polymeric shell [196]. Indeed, Skirtach et al. [197] used Au and Au sulphide NPs for modification of polymer capsule wall. Activation of capsules was achieved using NIR-laser irradiation $(830 \mathrm{~nm})$ with the subsequence release of loaded fluorescent dye inside cancer cells [197]. Initial size of $\mathrm{Au}$ NPs was $15 \mathrm{~nm}$ in diameter, which has the maximum of plasmon band at approximately $520 \mathrm{~nm}$. However, the aggregation of $15 \mathrm{~nm}$ NPs results in red shift and broadening of plasmon band of Au NPs [198], which is more sufficient for NIR-light irradiation. In this study, fluorescence molecules were delivered into cytosol of individual A549 cell upon laser irradiation. The similar Au NP aggregates, embedded into the polymeric shell of capsules, were used for the in vivo release of adenosine triphosphate in order to activate Wnt signalling pathway in the small freshwater polyp Hydra vulgaris (Figure 11A) [199]. In another study, polymer capsules with outer silica layer were also modified with $\mathrm{Au} \mathrm{NP}$ aggregates in order to deliver $\mathrm{pH}$-sensitive 


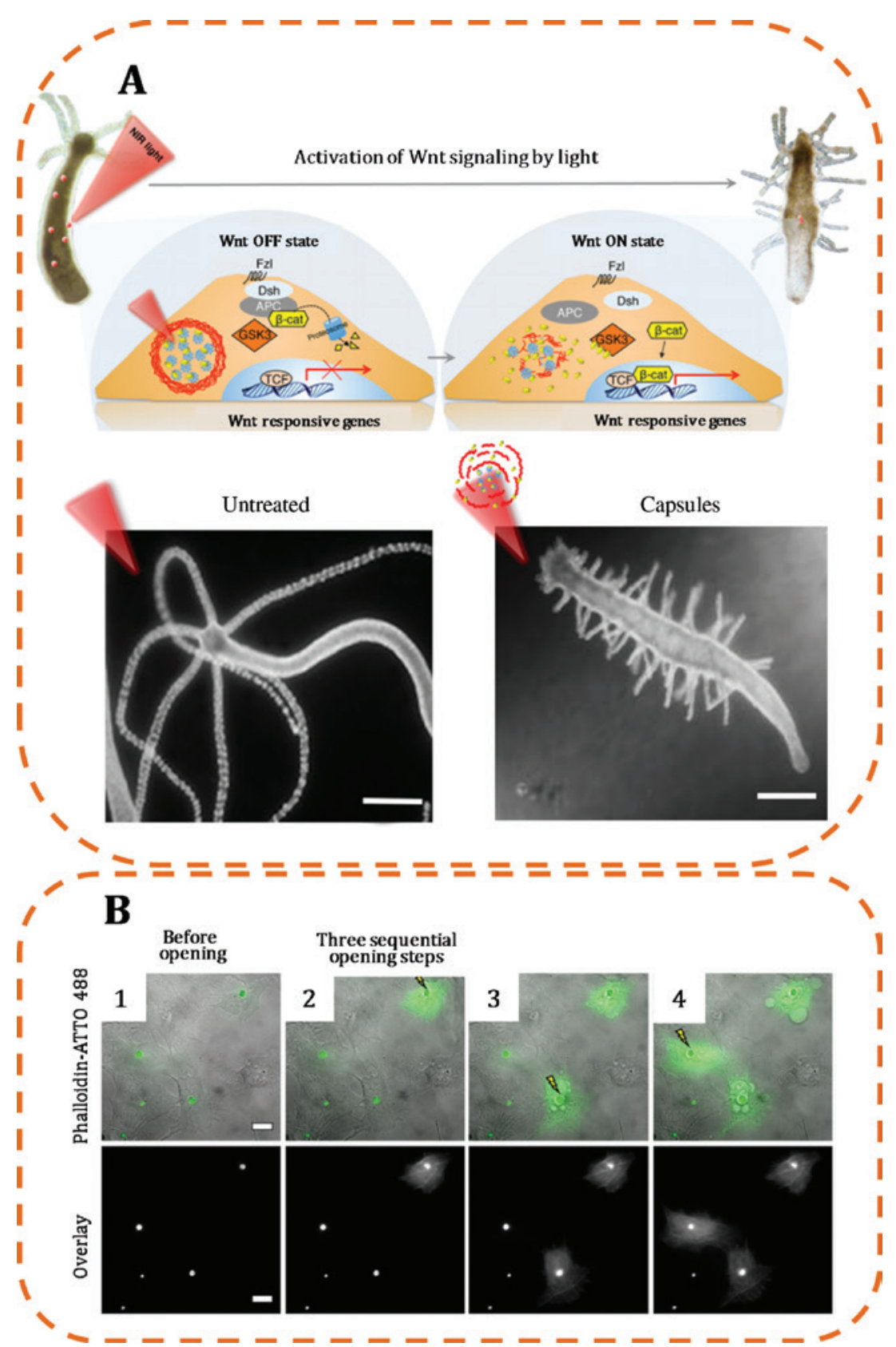

Figure 11: Light-induced release of bioactive compounds from composite polymer carriers.

(A) NIR-triggered activation of Wnt signalling pathway, (top) schematic diagram of experimental procedure, (left bottom) untreated animal, irradiated, (right bottom) animal treated with light-sensitive capsules and irradiated. Scale bars correspond to $500 \mu \mathrm{m}$. (B) Fluorescence and overlay (fluorescence + bright field channel) images of cells incubated with light-sensitive capsules. In (2-4), images are shown after three illumination steps. The scale bar corresponds to $20 \mu \mathrm{m}$. Reprinted from [9, 199]. Copyright 2016 American Chemical Society; Copyright 2018 by Wiley-VCH Verlag GmbH \& Co. KGaA, Weinheim.

fluorescent molecules upon laser irradiation. Additional layer was introduced to encapsulate low-molecular-weight cargo, which was not able to diffuse through the dense Si layer [200]. The synthesis procedure of polymer capsules with embedded $\mathrm{Au}$ aggregates cannot be fully controlled, as the induction of NP aggregates is a statistical process. Therefore, in this case, it is not always possible to achieve a good match of applied wavelength of laser irradiation with the maximum peak of the plasmon band. For this reason, other types of heating agents were applied to achieve more efficient NP heating at the same laser power density. In the recent study, Au nanostars with the narrow size distribution and the absorption maximum at approximately $800 \mathrm{~nm}$ were employed to functionalise polymeric 
capsules. Kantner et al. [9] demonstrated successful cellby-cell laser-induced staining, which were realised with polymer capsules modified with Au nanostars. As fluorescence markers, DAPI (4',6-diamidino-2-phenylindole), propidium iodide, phalloidin conjugated with dye, and wheat germ agglutinin conjugated with dye were used. Authors additionally performed intracellular staining of cells with polymer capsules loaded with different cargoes upon laser irradiation (Figure 11B) [9]. As an alternative to Au nanostars, Au nanorods can be used as heating agents in the polymer capsules wall, as Au nanorods possess two plasmon peaks (transversal and longitudinal) [201]. One of these peaks is situated in the NIR region, which is suitable for biomedical applications. In the recent work, polymer capsules modified with $\mathrm{Au}$ nanorods were employed to functionalise surface of scaffolds, which can be used as a frame for the new tissue formation. Capsules were loaded with hormone (dexamethasone), which in combination with $\beta$-glycerophosphate stimulates differentiation of mesenchymal stem cells (MSCs) in osteogenic direction in vitro. Burst-like release of bioactive compounds onto the scaffolds surface was probed using NIR-laser irradiation [202]. In recent studies, Au nanorods were also employed to induce noninvasive release of drugs from polymer capsules in vivo. Upon NIR irradiation at used laser power density $0.54 \mathrm{~J} \mathrm{~cm}^{-1}$ DOX was released inside bearing nude mice [203, 204]. Unusual shape of light-sensitive drug carriers was reported by $\mathrm{Wu}$ et al. [205], where polymer tubes were functionalised with Au NPs. Theoretical and experimental study demonstrated the explosion of developed systems upon laser irradiation. Authors claimed that mechanism of tube explosion is based on rapid evaporation of water inside tubes caused by photothermal effects [205]. Not only plasmonic NPs can be employed as nanoheaters into the capsules wall. Kurapati and Raichur [206] embedded GO into polymer capsules, which possesses superior optical absorption and photothermal conversion. Thus, GO-modified polymer capsules were also developed to be NIR light-sensitive [206]. Diversity of polymer capsules enables encapsulation of different nanostructures at the same time. For instance, Yashchenok et al. [207] incorporated Au NPs and single-walled carbon nanotubes (SWCNs) into the polymer capsule wall in order to perform thermometry at the nanoscale. Au NPs were employed as nanoheaters, and SWCNs as Raman nanothermometers. The obtained results demonstrated that upon NIR light irradiation the temperature of composite polymer capsule destruction was $348 \mathrm{~K}$ for a power density of $31.8 \times 10^{4} \mathrm{~W} \cdot \mathrm{cm}^{-2}$ [207]. It is worth mentioning that incorporation of nanostructured materials (e.g. plasmonic NPs) into the polymer capsule shell enables not only noninvasive light-induced drug delivery but also imaging of intracellular environment with e.g. photoacoustic imaging technique. This results in theranostic platform for an effective therapy and diagnosis [208, 209].

\subsection{Composite light-sensitive bioinspired delivery platforms}

Most of organic and inorganic drug carriers, which are responsive to light, do not reach clinical trials. Only some of them are officially approved by the Food and Drug Administration [24]. The main barrier between the development of the light-sensitive drug carriers and their translation into clinics is attributed to the ability of the organism to recognise and remove foreign biomaterial. Therefore, the novel design of the drug delivery platforms is required to protect the carriers from the cell immune system [24, 210]. Therefore, biomimetic-based approaches that attempt to imitate the real biological situation in cells and tissues are of great interest. Such delivery systems transfer specific biological functionalities to synthetic drug carriers [24, 211]. Light-responsive biological (bioinspired) drug carriers can be considered as one of the most attractive delivery systems, replicating features that are similar to the biological objects [212]. The main advantages of bioinspired carriers are their ability to interact with specific sites of the body, their cell/tissue entering mechanisms, capability to avoid the immune responses, and prolonged circulation times in biological fluids [212, 213]. Light-responsive bioinspired carriers include the use of (4.2.1) mammalian cells, (4.2.2) bacteria, and (4.2.3) viruses (Figure 12).

\subsubsection{Cell-based drug carriers}

Cell-based delivery systems attract tremendous attention because of the treatment ability of various diseases including oncology, diabetes, hemophilia, and cardiomyopathy. At the same time, cell engineering in combination with the designed nanoparticles and microparticles enables fabrication of biomimetic light-responsive platforms for the delivery of different bioactive compounds. Among different therapeutically relevant cells, RBCs, immune cells, SCs are widely employed as bioinspired drug delivery platforms and will be further discussed in details [214, 215].

\subsubsection{Red blood cells}

After in vivo administration, therapeutic formulations rely predominantly on the vascular system for the effective 
BIO-INSPIRED I.IGIIT-RESPONSIBI.E CARRIIRS

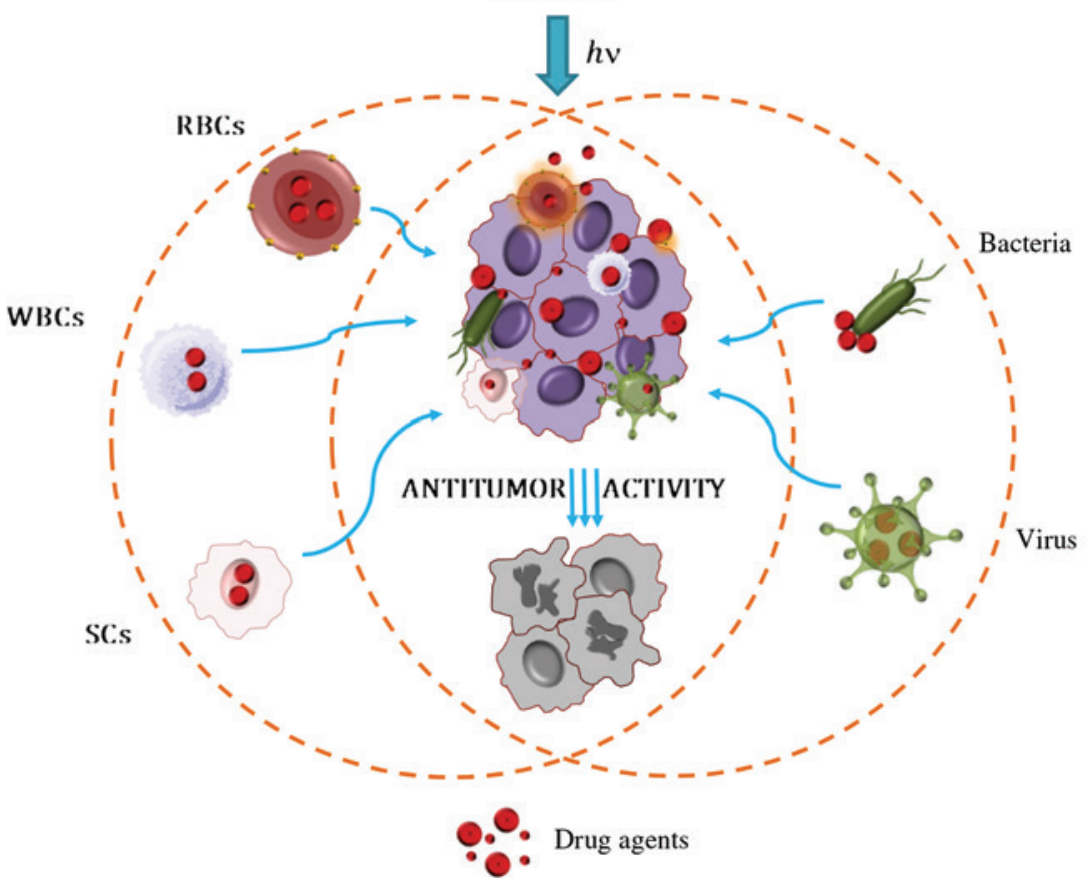

Figure 12: Schematic illustration of implementation of bioinspired light-sensitive carriers.

biodistribution and accumulation at the target site. RBCs or erythrocytes are the most abundant cells in the human blood system (approximately 99\% of the total amount of blood cells). RBCs have an average lifespan of 120 days without being cleared by macrophages. In comparison, the lifespan of the PEGylated drug carriers, which is known to have prolonged circulation times, is approximately $10 \mathrm{~h}$ [216, 217]. RBCs have a diameter of $7-8 \mu \mathrm{m}$ and an average volume of $90 \mathrm{fl} \mathrm{oz}$; they lack a nucleus and can change their shape to fit through the blood vessels. Erythrocytes confine the unintended extravasation and extend the circulation time in the body, which in combination with high cargo loading capacity, excellent biocompatibility, and low immunogenicity makes them as excellent drug delivery systems [216, 218]. RBCs can be reversibly opened under hypotonic conditions to inject exogenous compounds upon resealing of membrane pores. Another method of RBC drug loading involves the attachment of drug molecules to the cell surface through biotin, affinity ligands, or nonspecific interactions such as van der Waals forces, hydrogen bonding, or electrostatic or hydrophobic forces [215, 216].

For the controlled therapy, the RBC membranes can be modified with light-responsible agents. Delcea et al. [219] reported on RBCs modified with aggregates of Au NPs with high NIR absorbance. The attachment of Au NPs to RBCs was verified with Raman spectroscopy. The mechanism of the drug release was the heating of the lipid bilayer and/or transmembrane proteins. The developed system demonstrated improved drug release from the RBC biocarriers upon NIR-laser irradiation. Gao et al. [220] developed a cell-based delivery system composed of RBCs modified with the PS chlorin e6, which was incorporated into the membrane of erythrocytes upon mixing without disturbing the membrane structure. To achieve chemotherapeutic effect, DOX was introduced into the inner aqueous cavities of RBCs. The mechanism of photoinduced release of the drug was the break of cells' membrane upon laser irradiation. The resulted drug delivery platform possessed synergetic therapeutic effect from generation of ROS (PDT) and chemotherapy upon exposure to the 660-nm light [220]. However, the RBC-based delivery systems have some limitations. The main disadvantage of the RBC implementation as drug carriers is their ex vivo modifications including hemotransfusion (transferring of blood or blood products intravenously), which limit their wide application. Another challenge of the RBC employment as delivery carriers is their loading with drugs, which can sometimes lead to the change of their structural and functional properties [216, 221].

\subsubsection{Immune cells}

Another promising type of cell-based drug delivery platform is immune cells or white blood cells (WBCs). The 
main feature of immune cells is the response to injuries. Damage tissues produce signalling molecules such as chemokines and cytokines, which attract immune cells that infiltrate into the damage sites, e.g. infection, inflammation, tissue injury, and cancer. Immune cells are capable of deep penetration into tissues compared to the conventional drugs [222, 223]. The size of WBCs varies from $7 \mu \mathrm{m}$ (small lymphocytes) to $20 \mu \mathrm{m}$ (monocyte) [224]. The WBCs derived from the body can be loaded in vitro with drugs without any apparent toxic effects and reinjected into the blood. Immune cells can be also modified with light-responsive agents for the noninvasive therapy of cancer [225, 226].

There are different types of WBCs (leukocytes) such as neutrophils, lymphocytes, and macrophages, which can be employed as cell-based drug delivery vehicles to mediate the delivery across the biological barriers. The modification of leukocytes with light-responsive agents enables the release the drugs to targeted sites upon laser irradiation. The key feature of leukocytes is their migration abilities towards inflammatory area and adhesion to endothelial wall tissues with tumour cells [212, 223, 227].

Neutrophils present the most abundant leukocyte in the human organism. They are the first cell type that appears at the damage sites and secrete cytokines to recruit other cells. Neutrophils have a short lifespan (approximately 5 days) in blood circulation and only a few hours after isolation from the body. On the one hand, their shorter lifespan limits neutrophil applications in drug delivery. However, their ability to be instantly recruited makes these cells attractive as drug vehicles [228]. Chu et al. [229] showed a drug delivery system based on fluorescent polystyrene NPs coated with anti-CD11b antibodies via biotin-neutravidin binding that target activated neutrophils. The migration of the developed platforms was induced by neutrophils with the following infiltration into tumour. To achieve PTT, neutrophils were decorated with Au NPs. The neutrophil uptake of NPs did not alter neutrophil activation and transmigration. The developed cell-based delivery system possessed features of combined PTT and PDT under $660 \mathrm{~nm}$ light [229].

Lymphocytes are primarily presented in the blood circulation and lymphoid organs (spleen, tonsils, lymph nodes). $\mathrm{T}$ and $\mathrm{B}$ cells are the main types of lymphocytes that are responsible for the adaptive immune system. $\mathrm{T}$ cells play a key role in cell-mediated immunity, whereas $\mathrm{B}$ cells are involved in humoural immunity. Both $\mathrm{T}$ and $\mathrm{B}$ cells present multiple functions in the organism and are involved in numerous processes such as detecting antigens, infiltrating damage tissues, and attacking abnormal cells [228]. The advantages of drug delivery by lymphocytes are their migration and accumulation inside the tumour areas. Moreover, such delivery system can detect and attack metastases that are hard to be diagnosed $[215,230]$. Another type of lymphocytes, socalled NK cells, can more effectively attack infections and tumours [231]. They constitute approximately $10 \%$ of circulating lymphocytes and, unlike T lymphocytes, can eliminate target cells spontaneously without antigenspecific stimulation. Deng et al. [232] designed NK cell membranes decorated with NPs loaded with PSs. Photosensitizers $\left[4,4^{\prime}, 4^{\prime \prime}, 4^{\prime \prime \prime}\right.$-(porphine-5,10,15,20-tetrayl) tetrakis (benzoic acid)] loaded into NK carriers enhanced NK cell immunotherapy and eradicated primary tumour cells through PDT [232].

Macrophages are mononuclear phagocytes that play a crucial role in eliminating pathogens and cellular debris. They can act as antigen-presenting cells and secrete various types of bioactive compounds to communicate with other immune cells [233]. Macrophages can engulf different molecules based on specific receptor interactions and their phagocytic capabilities [216]. Choi et al. [234] used macrophages to deliver Au nanoshells to the tumour hypoxic regions. After application of NIR light, cancer cells were destroyed within these tumour areas [234]. Other works demonstrated the migratory potential of drugloaded macrophages in glioma both in vitro (tumour spheroids) and in vivo (mice models) [225, 235]. In the first work, $\mathrm{Au}$ nanoshells were used to modify macrophages and to suppress tumour spheroid growth upon NIR-laser irradiation. As the NPs are not able to cross the patent blood-brain barrier, macrophages can overcome this limitation. In the second work, authors demonstrated that macrophages readily traverse the blood-brain barrier, where residual glioma cells were found after surgery. Macrophages have the potential to increase NP delivery to different types of tumours including glioblastoma multiforme [225].

Overall, immune cells have a quick response and intrinsic homing properties with respect to damaged tissues and tumours. The sensitive detection of target sites and abilities to overcome biological barriers can facilitate drug delivery. However, among the available immune cells, leukocytes are difficult to cultivate and handle. They have relatively short lifespans, which hinder the manipulation processes for the drug loading [215].

\subsubsection{Stem cells}

Stem cells, in particular, MSCs, have attracted great interest because of their properties of intrinsic inflammation and tumour tropism. Because of the property of active migration towards tumours and penetration tumours, SCs 


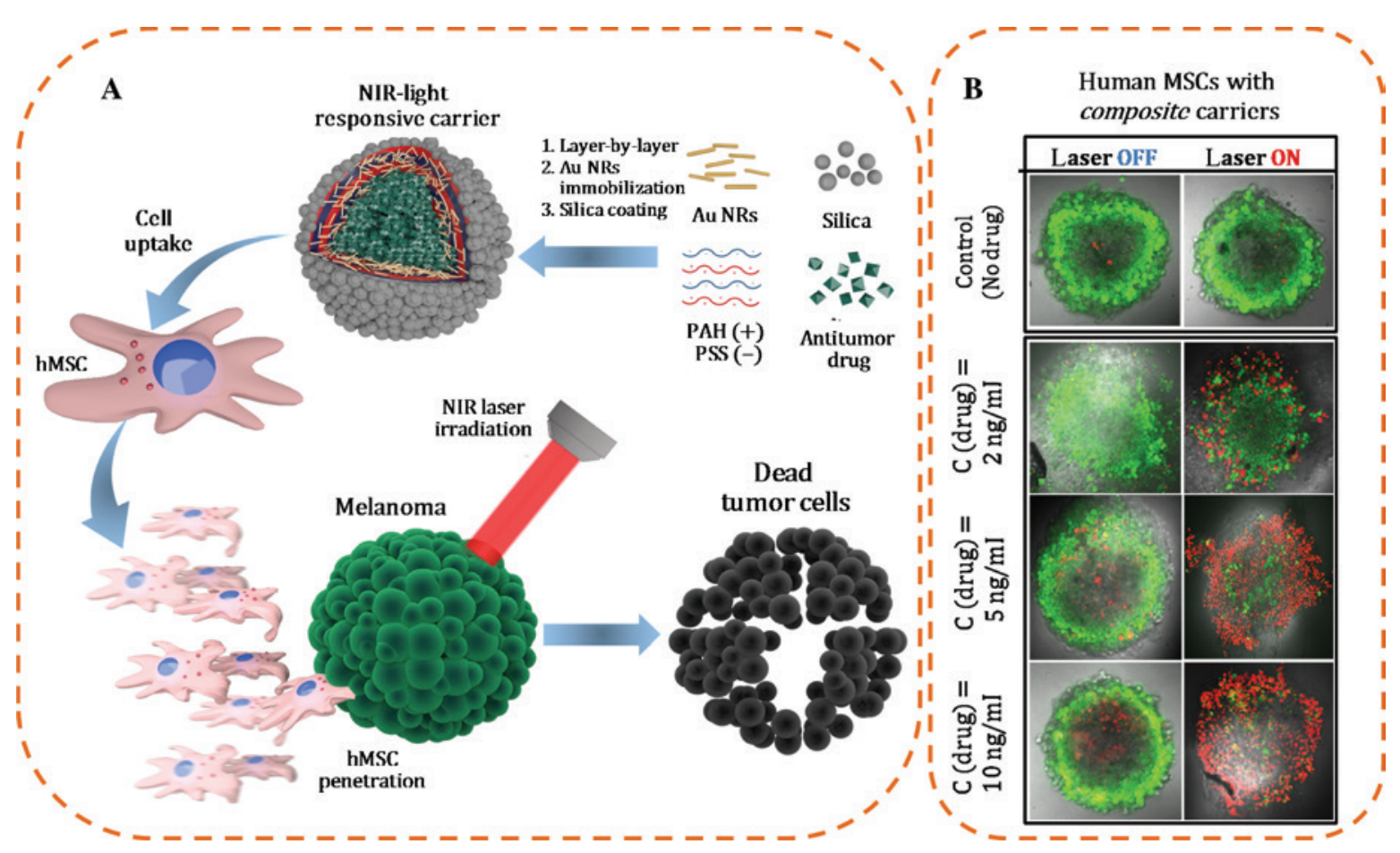

Figure 13: Addressable drug delivery using light-sensitive MSCs-based delivery platform.

(A) Schematic diagram of the experimental procedure. (B) Confocal microscopy images of tumour spheroids with and without NIR-laser irradiation incubated with (left) free antitumour drug, (middle) polymer light-sensitive particles loaded with antitumour drug, (right) MSCmodified polymer light-sensitive particles loaded with antitumour drug. Living cells were stained with calcein AM (green); nuclei of dead cells were stained with propidium iodide (red). Scale bars correspond to $100 \mu \mathrm{m}$. Reprinted with permission from [241]. Copyright 2019 Royal Society of Chemistry.

are applied as bioinspired carriers with wide possibilities of drug loading such as apoptosis-inducing and antiangiogenic factors, cytotoxic chemotherapy, immunomodulatory agents, oncolytic viruses, drug-loaded microparticles and NPs, and tissue- or tumour-specific prodrugs [236, 237]. The cytokine release in the tumour stroma and the interaction of MSCs with cytokine and chemokine receptors present on the cell surfaces are mainly responsible for their migration towards tumours [238, 239]. Xu et al. [240] developed a synergistic treatment platform based on MSCs loaded with light-responsive plasmonic-magnetic hybrid NPs containing lipids, DOX, Au nanorods, and $\mathrm{Fe}_{3} \mathrm{O}_{4}$ nanocluster (MSCs-LDGI) for photoacoustic imaging, targeted PTT, and chemotherapy against triple-negative breast cancer. Mesenchymal stem cells-LDGI maintained good bioactivity to migrate towards cancer cells both in vitro and in vivo. The disassembly of the developed nanostructures with the subsequence drug release was probed upon light irradiation. In vitro and in vivo experiments showed that the MSCs-LDGI efficiently inhibited cancer cell growth and implemented good therapeutic effects via both local and intravenous injection routes [240]. In recent work, Muslimov et al. [241] developed the light-sensitive MSCsbased delivery system. Antitumour drug was encapsulated into composite capsules functionalised with Au nanorods. In turn, capsules were used to modify MSCs. Upon NIRlaser irradiation, composite capsules were disintegrated because of the thermal effects. The efficiency of the developed MSC-based delivery system was probed on model of melanoma spheroids. NIR-triggered drug release indicated the higher antitumour efficiency of bioinspired platform compared to the free drug (Figure 13) [241].

\subsubsection{Bacteria-based drug carriers}

Pathogens such as bacteria have developed unique strategies to avoid the immune response and induce favourable interactions with host target cells [71]. Bacteria can penetrate even nonphagocytic mammalian cells by specific surface molecules. Bacteria can successfully grow in tumour sites because of the impaired blood circulation and large necrosis area of solid tumours [242]. Bacteria have the full complement of ribonucleic acid (RNA) polymerases, and they are able to deliver or produce these proteins at the target site [243]. Bacteria-based engineering strategies for drug delivery include recombinant bacteria, microbots, and bacterial ghosts. 
Recombinant bacteria are carriers that are genetically modified by the expression systems encoding proteins and antigens [71]. Hosseinidoust et al. [243] developed an optogenetic and light-sensitive delivery system based on recombinant bacteria using an engineered bacterial lightoxygen-voltage protein that bonded DNA when illuminated with blue light. The system showed a high dynamic range of protein expression, rapid activation, and deactivation kinetics in vitro. The recombinant bacteria were employed to activate transcription in different eukaryotic systems (mammalian cell lines and zebrafish embryos) upon stimulation with blue light [243-245].

Microbots are bacteria that transfer drug agents attached onto their surface. Microbots do not require genetic modifications for the drug delivery. This approach employs the invasive properties of bacteria that are able to migrate into the tumours, which cannot be reached by conventional chemotherapy. The genetically unmodified bacteria can be conjugated with the NPs via biotinstreptavidin interactions or different chemical-labile linkers [246] (Figure 14A). Luo et al. [248] reported on the bacteria-mediated targeting hypoxia (oxygen reduced tumour) to offer the expandable spectra for cancer theranostics. The authors developed two drug delivery approaches including a cargo-carrying method and an antibody-directed method. These approaches allowed delivering upconversion nanorods for imaging and $\mathrm{Au}$ nanorods for PTT ablation upon NIR light excitation. The antibody-directed strategy demonstrated more effective treatment giving high-level bioimaging, longer retention period, and effective therapy of tumours [248].

Bacterial ghosts are empty cell envelopes of Gram-negative bacteria. In bacterial ghosts, plasma components with all genetic material are removed. They can be produced by controlled expression of the cloned lysis gene $\mathrm{E}$ from bacteriophage species, generating a lysis tunnel structure within the envelope of the living bacteria [249]. Bacterial ghosts keep cellular morphology similar to native bacteria, where the entire surface structures including adhesins, membrane proteins, lipopolysaccharides, and the peptidoglycan layer are preserved [250]. Drug carriers can be also obtained from bacterial outer membrane vesicles [251]. Gujrati et al.

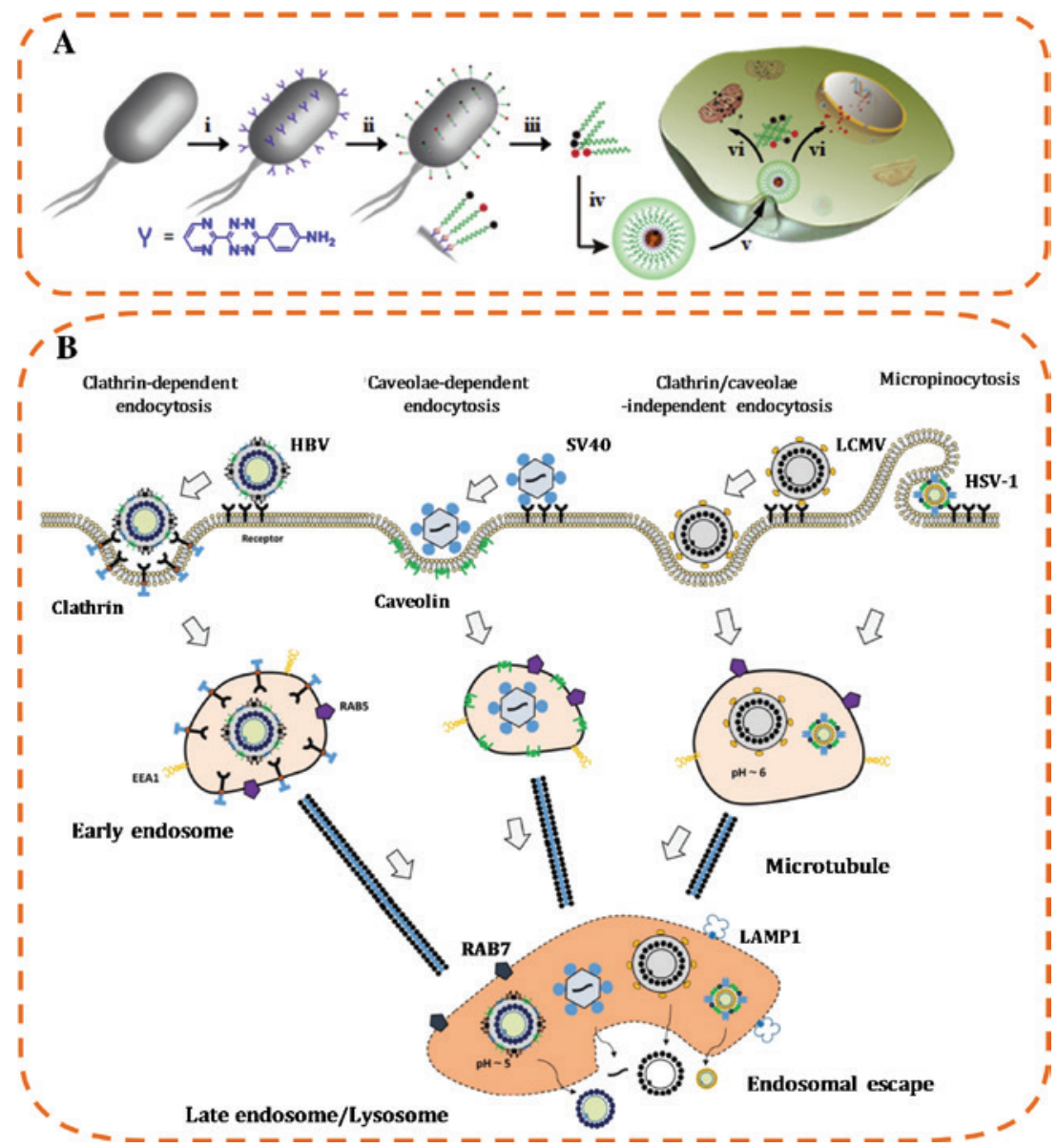

Figure 14: Drug delivery using light-sensitive bacteria and viruses.

(A) Schematic diagram of the bacterial microbots to load, deliver, and release active drugs for cancer therapy. (B) Cellular uptake mechanisms of viruses. Reprinted with permission from [246, 247]. Copyright 2018 Elsevier, Copyright 2017 Ivyspring. 
[252] developed bioengineered bacteria vesicles for both contrast enhancement in photoacoustic imaging and PTT. These bacterial vesicles demonstrated several advantages, as they possessed a rigid membrane with high stability and reduced leakage in the systemic circulation. The authors obtained bacteria vesicles with encapsulated melanin using an Escherichia coli strain. This bacterial strain was previously modified to be less endotoxic and to overexpress tyrosinase, which produces melanin that is passively incorporated into the cytosol and membrane of vesicles. The obtained results indicated that upon NIR light irradiation bacteria vesicles with melanin generated strong optoacoustic signals appropriate for bioimaging applications. In addition, the developed bacteria vesicles released heat upon NIR-laser irradiation, which resulted in photothermal effects both in vitro and in vivo [252].

\subsubsection{Viruses-based drug carriers}

Viruses are infectious microorganisms consisting of nucleic acids in a protein coating (capsid). Viruses possess several properties that can be effectively used for the development of bioinspired drug delivery carriers. As previously discussed, an ideal drug carrier should avoid host cell immune response, and target cells of interest with further release of the loaded cargo into the cytoplasm. These processes fully describe the early stage of the virus infection. Viruses naturally evolved to deliver genetic material into target cells. Moreover, viruses can evade host body protection and reach specific tissues or cells. Once viruses are taken up by host cells, they colonise the intracellular space and release the DNA or RNA to replicate themselves (Figure 14B). Viruses utilise their protein components to overcome cellular barriers. Drug delivery technologies can learn a great deal from viruses [247].

Viral vectors were one of the first systems to deliver specific genes. Such vectors included inactivated adenoviruses, adeno-associated viruses, retroviruses, and lentiviruses. Viral vectors had to transfer only the required genes to the target cells. For this, they were modified to minimise the risk of their expansion. The process of inactivation involved the deletion of a part of the viral genome critical for the viral replication. Such vectors can infect cells but are not able to produce the new virions without a helper virus [24, 253,254]. Viral vectors can be also modified for an effective PTT. Everts et al. [255] covalently bound adenoviral vectors with $\mathrm{Au}$ NPs, so that the developed engineered systems successfully retargeted a tumour-associated carcinoembryonic antigen without losing the viral vector infectivity. Jung et al. [256] explored the combination of oncolytic adenovirus and $\mathrm{Au}$ nanorod-mediated hyperthermia to improve antitumour effect against head and neck cancer. Exposure of the virus-Au nanorods system to light improved endocytosis of oncolytic adenovirus, transgene expression, and subsequent cytolysis of head and neck cancer cells [256]. In another study, Pandori et al. developed light-responsive viruses-based delivery system by conjugating photocleavable moieties to viral components, which possessed no infectivity. The exposure of the modified viruses to the UV light induced the modification of photocleavable moieties, which resulted in restoration of the virus infectivity [257].

\section{Conclusion and outlook}

Light-responsive nanostructured materials are successfully employed in the field of nanomedicine for the delivery of drugs. Given the nature of particles, different mechanisms can be employed for the release of bioactive compounds. In this review article, we considered two major groups of light-sensitive carriers, plain and composite. Each group consisted of either plasmonics and dielectric NPs, or organic and bioinspired drug carriers.

\subsection{Plasmonic NPs}

As discussed above, plasmonic NPs ( $\mathrm{Au}$ and $\mathrm{Ag}$ ) and their combination open up a great potential for the development of optically responsive drug delivery platforms. The rational design and fabrication of anisotropic $\mathrm{Ag}$ and $\mathrm{Au}$ NPs with different morphology (e.g. shape and size) provide various strategies for light-induced cancer treatment. The fundamental, careful, and accurate investigations of $\mathrm{Ag} / \mathrm{Au} \mathrm{NP}$ morphology on cellular toxicity and behaviour are highly demanded. Also, chemical stability of Ag or Au NPs in biological fluids is required. The surface coating of $\mathrm{Au} / \mathrm{Ag}$ NPs with biopolymers or proteins significantly increases their application in medicine for drug delivery. At present, the main stream in application of plasmonic NPs as drug carriers is devoted to the development of combinational Au- or Ag-based nanocarriers.

\subsection{Dielectric NPs}

Compared to plasmonic NPs, dielectric NPs are not widely used as drug delivery carriers. However, similar to plasmonic NPs, Si NPs are excellent nanoheaters, and based 
on their size and shape, they can be excited in the widerange spectrum [26]. This feature of Si NPs in combination with an effective field enhancement [123] can be successfully used in PTT of malignant neoplasms with simultaneous visualisation of NP biodistribution in vivo with optical fluorescence microscopy. For this, Si NPs should be modified with fluorescence molecules, which requires additional synthetic steps. Bioimaging of $\mathrm{TiO}_{2} \mathrm{NPs}$ is also not straightforward, and it requires additional surface modification. With the progress in material science, the limitations of the employment of UV light for biological tissues to induce generation of ROS can be overcome by the doping/modification of $\mathrm{TiO}_{2} \mathrm{NPs}$ with other materials (rare earth, magnetic); therefore, irradiation wavelength can be shifted to areas with lower energies. Iron oxide NPs can also act as nanoheaters, which in combination with external alternating magnetic field (AMF) significantly improves PTT providing synergetic effect from magnetic field and light energy. Upconverting NPs are currently under an extensive investigation, as they are nonphototoxic with wide surface functionalisation possibilities. However, there are some limitations that still need to be improved. First is the relatively low quantum yield of the converted light, which requires higher power excitation. Moreover, stability of lanthanide dopants, as well as photoresponsive moieties, needs to be further investigated prior to in vivo applications. All discussed types of dielectric NPs are promising drug delivery carriers with certain benefits and drawbacks; however, IONPs are closer to the real clinical applications, as this material is clinically approved, and clinical trials on humans were already conducted $[258,259]$.

\subsection{Composite delivery systems based on organic NPs}

To combine therapeutic and diagnostic properties in one carrier, composite delivery systems are under intensive investigation. Indeed, composite organic NPs are often utilised to optimise delivery of bioactive compounds, to prolong circulation time, to enable bioimaging, and to provide combined therapy (e.g. chemotherapy and PDT). Easy modification of micelles and liposomes with lightsensitive moieties enables controllable drug release upon laser irradiation. However, prior to complete implementation of micelles and liposomes in clinics, some issues should be addressed. First, biodegradability and biocompatibility of these carriers, as well as their products after photoreaction, should be improved. Another issue that needs to be solved is NIR-sensitive micelles and liposomes that are of urgent need. For this, multiphotonreaction mechanisms are required, which will result in a wider application of micelles and liposomes in nanomedicine. Despite biocompatibility, easy synthesis, wide functionalisation possibilities, polymer capsules are also still not introduced into clinics because of the short circulation time and large size, which are not always optimal for in vivo applications. Therefore, light-sensitive bioinspired drug delivery platforms can help to overcome the abovementioned limitations of composite carriers based on organic polymers.

\subsection{Bioinspired delivery platforms}

These delivery systems based on cells, bacteria, or viruses loaded with the pharmaceuticals (or contrast agents for imaging) retain their biological functions: their distribution will mimic their natural movement to the disease areas. However, despite all the advantages of light-responsive bioinspired delivery platforms, large size of cells can be a significant physical barrier that prevents complete dispersion of these cells in small vessels of the vascular network. This fact limits access of exogenously introduced cells to many target sites, including tumour tissues [236, 240]. Despite the advantages of discussed bacteria-based drug systems, there are several challenges in their development and utilisation. First, the toxicity of bacterial drug carriers still remains a serious limitation. The bacteriainduced toxicity at the therapeutic efficacy dose can cause a rapid bacteria clearance or autoimmune response. The second challenge is drug loading. Loading of agents with average sizes of hundreds nanometers affects the motion of bacteria, diffusion from blood vessels, and migration into tumours. The third concern is the lack of tissue distribution profile of bacterial carriers. Moreover, anaerobic bacteria can selectively infect and break hypoxic regions but leave a well-oxygenated outer rim of the tumours that can lead to tumour regrowth [242, 246]. The viruses-based delivery systems also suffer from limitations such as a potential immunogenicity, pathogenicity, and the broad tropism of viruses.

\subsection{Future perspectives}

Nevertheless, it seems that the development of composite light-responsive carriers consisting of individual parts of organic and inorganic nature is the direction of future research in the field of nanomedicine. This will make it possible to take advantage of each component of 
the developed smart delivery platform and enable, e.g. enhanced loading with drugs, multimodal imaging, targeted properties, prolonged circulation times in blood, and so forth. Moreover, the application of light-sensitive composite carriers will be able to provide noninvasive activation of therapeutics in combination with PTT at the target sites in vivo.

\section{Abbreviations}

\begin{tabular}{|c|c|}
\hline${ }^{1} \mathrm{O}_{2}$ & singlet oxygen \\
\hline${ }^{3} \mathrm{O}_{2}$ & molecular oxygen \\
\hline Ag NPs & silver nanoparticles \\
\hline $\mathrm{Au}$ NPs & gold nanoparticles \\
\hline $\mathrm{AZO}$ & azobenzene \\
\hline BSA & bovine serum albumin \\
\hline CT-26 & murine colon carcinoma \\
\hline CW & continuous wave \\
\hline DAPI & 4',6-diamidino-2-phenylindole \\
\hline DNA & deoxyribonucleic acid \\
\hline DNQ & 2-diazo-1,2-naphthoquinone \\
\hline DOX & doxorubicin \\
\hline DPPC & 1,2-dipalmitoyl-sn-glycero-3-phosphocholine \\
\hline DSPC & 1,2-distearoyl-sn-glycero-3-phosphocholine \\
\hline GO & graphene oxide \\
\hline HA & hyaluronic acid \\
\hline IONPs & iron oxide nanoparticles \\
\hline IR & infrared \\
\hline $\begin{array}{l}\text { LCST } \\
\text { micelles }\end{array}$ & $\begin{array}{l}\text { lower critical solution temperature } \\
\text { amphiphilic macromolecules }\end{array}$ \\
\hline MRI & magnetic resonance imaging \\
\hline MSCs & mesenchymal stem cells \\
\hline NB & $o$-nitrobenzyl \\
\hline NIR & near-infrared \\
\hline NK cells & natural killer cells \\
\hline NP & nanoparticle \\
\hline PDT & photodynamic therapy \\
\hline PEG & polyethylene glycol \\
\hline PS & photosensitizer \\
\hline PTT & photothermal therapy \\
\hline QDs & quantum dots \\
\hline RBCs & red blood cells \\
\hline RNA & ribonucleic acids \\
\hline ROS & reactive oxygen species \\
\hline RT & room temperature \\
\hline $\mathrm{Ru}$ & ruthenium \\
\hline SCs & stem cells \\
\hline SERS & surface enhanced Raman spectroscopy \\
\hline Si NPs & silicon-based NPs \\
\hline SP & spiropyran \\
\hline SPACE & skin-penetrating-and-cell-entering \\
\hline SPR & surface plasmon resonance \\
\hline SWCN & single-walled carbon nanotubes \\
\hline TEM & transmission electron microscopy \\
\hline $\mathrm{TiO}_{2} \mathrm{NPs}$ & titanium dioxide nanoparticles \\
\hline TPE & two-photon excitation \\
\hline
\end{tabular}

UCNPs upconverting nanoparticles

UV ultraviolet

WBCs white blood cells

Acknowledgements: The part of this work related to the physical principles of light interaction with NPs was supported by the Ministry of Education and Science of Russian Federation (project 2.2267.2017/4.6). The part of this work related to the chemical mechanisms of light interaction with delivery carriers was supported by the Russian Foundation for Basic Research (project 18-3220089). The part of the work about bioinspired delivery platforms was supported by a grant from the Russian Science Foundation (project 19-75-10008). M.V.Z. thanks the President's Scholarship SP-1576.2018.4.

\section{References}

[1] Cai X, Luo Y, Zhang W, Du D, Lin Y. pH-sensitive ZnO quantum dots-doxorubicin nanoparticles for lung cancer targeted drug delivery. ACS Appl Mater Interfaces 2016;8:22442-50.

[2] Wang B, Han Y, Lin Q, Liu H, Nan K, Chen H. In vitro and in vivo evaluation of xanthan gum-succinic anhydride hydrogels for ionic strength-sensitive release of antibacterial agents. J Mater Chem B 2012;12:9.

[3] Liu F, Kozlovskaya V, Medipelli S, et al. Temperature-sensitive polymersomes for controlled delivery of anticancer drugs. Chem Mater 2015;27:7945-56.

[4] Yang C, Wang X, Yao X, Zhang Y, Wu W, Jiang X. Hyaluronic acid nanogels with enzyme-sensitive cross-linking group for drug delivery. J Control Release 2015;205:206-17.

[5] Skirtach AG, Yashchenok AM, Möhwald H. Encapsulation, release and applications of $L b L$ polyelectrolyte multilayer capsules. Chem Commun 2011;47:12736-46.

[6] Paris JL, Cabañas MV, Manzano M, Vallet-Regí M. Polymergrafted mesoporous silica nanoparticles as ultrasound-responsive drug carriers. ACS Nano 2015;9:11023-33.

[7] Qin Y, Chen J, Bi Y, et al. Near-infrared light remote-controlled intracellular anti-cancer drug delivery using thermo/pH sensitive nanovehicle. Acta Biomater 2015;17:201-9.

[8] Kuo C-Y, Liu T-Y, Chan T-Y, et al. Magnetically triggered nanovehicles for controlled drug release as a colorectal cancer therapy. Colloids Surf B Biointerfaces 2016;140:567-73.

[9] Kantner K, Rejman J, Kraft KVL, et al. Laterally and temporally controlled intracellular staining by light-triggered release of encapsulated fluorescent markers. Chem Eur J 2018;24:2098-102.

[10] Ash C, Dubec M, Donne K, Bashford T. Effect of wavelength and beam width on penetration in light-tissue interaction using computational methods. Lasers Med Sci 2017;32:1909-18.

[11] Liu G-Y, Chen C-J, Li D-D, Wang S-S, Ji J. Near-infrared light-sensitive micelles for enhanced intracellular drug delivery. J Mater Chem 2012;22:16865-71.

[12] Yan Q, Han D, Zhao Y. Main-chain photoresponsive polymers with controlled location of light-cleavable units, from 
synthetic strategies to structural engineering. Polym Chem 2013;4:5026-37.

[13] Bédard MF, Sadasivan S, Sukhorukov GB, Skirtach A. Assembling polyelectrolytes and porphyrins into hollow capsules with laser-responsive oxidative properties. J Mater Chem 2009;19:2226-33.

[14] Qiu M, Wang D, Liang W, et al. Novel concept of the smart NIRlight-controlled drug release of black phosphorus nanostructure for cancer therapy. Proc Natl Acad Sci 2018;115:501-6.

[15] Goodman AM, Hogan NJ, Gottheim S, Li C, Clare SE, Halas NJ. Understanding resonant light-triggered DNA release from plasmonic nanoparticles. ACS Nano 2017;11:171-9.

[16] Ke PC, Lin S, Parak WJ, Davis TP, Caruso F. A decade of the protein corona. ACS Nano 2017;11:11773-6.

[17] Hühn J, Fedeli C, Zhang Q, et al. Dissociation coefficients of protein adsorption to nanoparticles as quantitative metrics for description of the protein corona: a comparison of experimental techniques and methodological relevance. Int J Biochem Cell Biol 2016;75:148-61.

[18] Zyuzin MV, Yan Y, Hartmann R, et al. Role of the protein corona derived from human plasma in cellular interactions between nanoporous human serum albumin particles and endothelial cells. Bioconjug Chem 2017;28:2062-8.

[19] Zyuzin MV, Honold T, Carregal-Romero S, Kantner K, Karg M, Parak WJ. Influence of temperature on the colloidal stability of polymer-coated gold nanoparticles in cell culture media. Small 2016;12:1723-31.

[20] Wu B, Zhao M, Zhou J, Xu X, Wang C. Numerical investigation of nonlinear photothermal effect in vanadium dioxide phasechange particles. Opt Commun 2018;427:184-9.

[21] Hussain SZ, Zyuzin MV, Hussain I, Parak W], Carregal-Romero S. Catalysis by multifunctional polyelectrolyte capsules. RSC Adv 2016;6:81569-77.

[22] Zyuzin MV, Timin AS, Sukhorukov GB. Multilayer capsules inside biological systems, state-of-the-art and open challenges. Langmuir 2019;35:4747-62.

[23] Ermakov A, Lim SH, Gorelik S, et al. Polyelectrolyte-graphene oxide multilayer composites for array of microchambers which are mechanically robust and responsive to NIR light. Macromol Rapid Commun 2019;40:1700868.

[24] Parodi A, Molinaro R, Sushnitha M, et al. Bio-inspired engineering of cell- and virus-like nanoparticles for drug delivery. Biomaterials 2017;147:155-68.

[25] Jauffred L, Samadi A, Klingberg H, Bendix PM, Oddershede LB. Plasmonic heating of nanostructures. Chem Rev 2019;119:8087-130.

[26] Zograf GP, Petrov MI, Zuev DA, et al. Resonant nonplasmonic nanoparticles for efficient temperature-feedback optical heating. Nano Lett 2017;17:2945-52.

[27] Govorov AO, Richardson HH. Generating heat with metal nanoparticles. Nano Today 2007;2:30-8.

[28] Baffou G, Quidant R. Thermo-plasmonics, using metallic nanostructures as nano-sources of heat. Laser Photonics Rev 2013;7:171-87.

[29] Danesi S, Gandolfi M, Carletti L, et al. Photo-induced heat generation in non-plasmonic nanoantennas. Phys Chem Chem Phys 2018;20:15307-15.

[30] Van Dijk MA, Tchebotareva AL, Orrit M, et al. Absorption and scattering microscopy of single metal nanoparticles. Phys Chem Chem Phys 2006;8:3486-95.
[31] Hlaing M, Gebear-Eigzabher B, Roa A, Marcano A, Radu D, Lai C-Y. Absorption and scattering cross-section extinction values of silver nanoparticles. Opt Mater 2016;58:439-44.

[32] Lee C, Hahn JW. Calculating the threshold energy of the pulsed laser sintering of silver and copper nanoparticles. J Opt Soc Korea 2016;20:601-6.

[33] Metwally K, Mensah S, Baffou G. Fluence threshold for photothermal bubble generation using plasmonic nanoparticles. J Phys Chem C 2015;119:28586-96.

[34] Bilankohi SM. Optical scattering and absorption characteristics of silver and silica/silver core/shell nanoparticles. Orient J Chem 2015;31:2259-63.

[35] Bilankohi SM. The simulation of the optical characteristics of platinum and platinum/silica nanoparticles. Orient J Chem 2015;31:293-7.

[36] Duncan B, Kim C, Rotello VM. Gold nanoparticle platforms as drug and biomacromolecule delivery systems. J Control Release 2010;148:122-7.

[37] Baffou G, Rigneault H. Femtosecond-pulsed optical heating of gold nanoparticles. Phys Rev B 2011;84:035415.

[38] Hu M, Hartland GV. Heat dissipation for Au particles in aqueous solution: relaxation time versus size. J Phys Chem B 2002;106:7029-33.

[39] Skirtach AG, Dejugnat C, Braun D, et al. The role of metal nanoparticles in remote release of encapsulated materials. Nano Lett 2005;5:1371-7.

[40] Volkov AN, Sevilla C, Zhigilei LV. Numerical modeling of short pulse laser interaction with Au nanoparticle surrounded by water. Appl Surf Sci 2007;253:6394-9.

[41] Lukianova-Hleb E, Hu Y, Latterini L, et al. Plasmonic nanobubbles as transient vapor nanobubbles generated around plasmonic nanoparticles. ACS Nano 2010;4:2109-23.

[42] Tribelsky MI, Miroshnichenko AE, Kivshar YS, Luk'yanchuk BS, Khokhlov AR. Laser pulse heating of spherical metal particles. Phys Rev X 2011;1:021024.

[43] Lalisse A, Tessier G, Plain J, Baffou G. Quantifying the efficiency of plasmonic materials for near-field enhancement and photothermal conversion. J Phys Chem C 2015;119:25518-28.

[44] Wu X, Ni Y, Zhu J, et al. Thermal transport across surfactant layers on gold nanorods in aqueous solution. ACS Appl Mater Interfaces 2016;8:10581-9.

[45] Zhang D, Gökce B, Barcikowski S. Laser synthesis and processing of colloids: fundamentals and applications. Chem Rev 2017;117:3990-4103.

[46] Angelsky OV, Bekshaev AY, Maksimyak PP, Maksimyak AP, Hanson SG, Kontush SM. Controllable generation and manipulation of micro-bubbles in water with absorptive colloid particles by CW laser radiation. Opt Express 2017;25:5232-43.

[47] Ogunyankin MO, Shin JE, Lapotko DO, Ferry VE, Zasadzinski JA. Optimizing the NIR fluence threshold for nanobubble generation by controlled synthesis of 10-40 nm hollow Gold Nanoshells. Adv Funct Mater 2018;28:1705272.

[48] Sarkar D, Kang P, Nielsen SO, Qin Z. Non-Arrhenius reactiondiffusion kinetics for protein inactivation over a large temperature range. ACS Nano 2019;13:8669-79.

[49] Baffou G, Quidant R, García de Abajo FJ. Nanoscale control of optical heating in complex plasmonic systems. ACS Nano 2010;4:709-16.

[50] Jain PK, Lee KS, El-Sayed IH, El-Sayed MA. Calculated absorption and scattering properties of gold nanoparticles of different 
size, shape, and composition: applications in biological imaging and biomedicine. J Phys Chem B 2006;110:7238-48.

[51] Zograf GP, Timin AS, Muslimov AP, et al. All-optical nanoscale heating and thermometry with resonant dielectric nanoparticles for photoinduced tumor treatment. Laser Photonics Rev 2020. DOI: 10.1002/lpor.201900082.

[52] Milichko VA, Zuev DA, Baranov DG, et al. Metal-dielectric nanocavity for real-time tracing molecular events with temperature feedback. Laser Photon Rev 2018;12:1700227.

[53] Dmitriev PA, Baranov GD, Milichko AV, et al. Resonant raman scattering from silicon nanoparticles enhanced by magnetic response. Nanoscale 2016;8:9721-6.

[54] Krasilin AA, Volodina K, Sukhova AA, et al. The conformation of bovine serum albumin adsorbed to the surface of single all-dielectric nanoparticles following light-induced heating. J Biophotonics 2018;11:e201700322.

[55] Mitsai E, Naffouti M, David T, et al. Si 1-x Ge x nanoantennas with a tailored raman response and light-to-heat conversion for advanced sensing applications. Nanoscale 2019;11:11634-41.

[56] Danesi S, Alessandri I. Using optical resonances to control heat generation and propagation in silicon nanostructures. Phys Chem Chem Phys 2019;21:11724-30.

[57] O'Farrell N, Houlton A, Horrocks BR. Silicon nanoparticles, applications in cell biology and medicine. Int J Nanomedicine 2006;1:451-72.

[58] Hertz H. Ueber einen einfluss des ultravioletten lichtes auf die electrische entladung. Ann Phys 1887;267:983-1000.

[59] Einstein A. Über einen die erzeugung und verwandlung des lichtes betreffenden heuristischen gesichtspunkt. Ann Phys 1905;322:132-48.

[60] Brongersma ML, Halas NJ, Nordlander P. Plasmon-induced hot carrier science and technology. Nat Nanotechnol 2015;10:25-34.

[61] Lee SE, Liu GL, Kim F, Lee LP. Remote optical switch for localized and selective control of gene interference. Nano Lett 2009;9:562-70.

[62] Xin H, Namgung B, Lee LP. Nanoplasmonic optical antennas for life sciences and medicine. Nat Rev Mater 2018;3:228-43.

[63] Rana S, Yeh Y-C, Rotello VM. Engineering the nanoparticle-protein interface: applications and possibilities. Curr Opin Chem Biol 2010;14:828-34.

[64] Jain PK, Qian W, El-Sayed MA. Ultrafast cooling of photoexcited electrons in gold nanoparticle - thiolated DNA conjugates involves the dissociation of the gold - thiol bond. J Am Chem Soc 2006;128:2426-33.

[65] Linic S, Aslam U, Boerigter C, Morabito M. Photochemical transformations on plasmonic metal nanoparticles. Nat Mater 2015;14:567-76.

[66] Adleman JR, Boyd DA, Goodwin DG, Psaltis D. Heterogenous catalysis mediated by plasmon heating. Nano Lett 2009;9:4417-23.

[67] Fasciani C, Alejo CJB, Grenier M, Netto-Ferreira JC, Scaiano JC. High-temperature organic reactions at room temperature using plasmon excitation, decomposition of dicumyl peroxide. Org Lett 2011;13:204-7.

[68] Hirsch LR, Stafford RJ, Bankson JA, et al. Nanoshell-mediated near-infrared thermal therapy of tumors under magnetic resonance guidance. Proc Natl Acad Sci 2003;100:13549-54.

[69] Stern JM, Stanfield J, Kabbani W, Hsieh J-T, Cadeddu JA. Selective prostate cancer thermal ablation with laser activated gold nanoshells. J Urol 2008;179:748-53.
[70] El-Sayed IH, Huang X, El-Sayed MA. Selective laser photo-thermal therapy of epithelial carcinoma using anti-EGFR antibody conjugated gold nanoparticles. Cancer Lett 2006;239:129-35.

[71] Yoo S, Hong S, Choi Y, Park J-H, Nam Y. Photothermal inhibition of neural activity with near-infrared-sensitive nanotransducers. ACS Nano 2014;8:8040-9.

[72] Nakatsuji H, Numata T, Morone N, et al. Thermosensitive ion channel activation in single neuronal cells by using surfaceengineered plasmonic nanoparticles. Angew Chem Int Ed 2015;54:11725-9.

[73] Avouris P, Walkup RE. Fundamental mechanisms of desorption and fragmentation induced by electronic transitions at surfaces. Annu Rev Phys Chem 1989;40:173-206.

[74] Linic S, Christopher P, Ingram DB. Plasmonic-metal nanostructures for efficient conversion of solar to chemical energy. Nat Mater 2011;10:911-21.

[75] Watanabe K, Menzel D, Nilius N, Freund H-J. Photochemistry on metal nanoparticles. Chem Rev 2006;106:4301-20.

[76] Sirelkhatim A, Mahmud S, Seeni A, et al. Review on zinc oxide nanoparticles, antibacterial activity and toxicity mechanism. Nano-Micro Lett 2015;7:219-42.

[77] Fu PP, Xia Q, Hwang H-M, Ray PC, Yu H. Mechanisms of nanotoxicity: generation of reactive oxygen species. J Food Drug Anal 2014;22:64-75.

[78] Raghupathi KR, Koodali RT, Manna AC. Size-dependent bacterial growth inhibition and mechanism of antibacterial activity of zinc oxide nanoparticles. Langmuir 2011;27:4020-8.

[79] He W, Kim H-K, Wamer WG, Melka D, Callahan JH, Yin J-J. Photogenerated charge carriers and reactive oxygen species in zno/au hybrid nanostructures with enhanced photocatalytic and antibacterial activity. J Am Chem Soc 2014;136:750-7.

[80] Zhang W, Li Y, Niu J, Chen Y. Photogeneration of reactive oxygen species on uncoated silver, gold, nickel, and silicon nanoparticles and their antibacterial effects. Langmuir 2013;29:4647-51.

[81] Thrall ES, Preska Steinberg A, Wu X, Brus LE. The role of photon energy and semiconductor substrate in the plasmon-mediated photooxidation of citrate by silver nanoparticles. J Phys Chem C 2013;117:26238-47.

[82] Christopher P, Xin H, Linic S. Visible-light-enhanced catalytic oxidation reactions on plasmonic silver nanostructures. Nat Chem 2011;3:467-72.

[83] Lee J, Mubeen S, Ji X, Stucky GD, Moskovits M. Plasmonic photoanodes for solar water splitting with visible light. Nano Lett 2012;12:5014-9.

[84] Zhou L, Swearer DF, Zhang C, et al. Quantifying hot carrier and thermal contributions in plasmonic photocatalysis. Science 2018;362:69-72.

[85] Bard AJ, Fox MA. Artificial photosynthesis, solar splitting of water to hydrogen and oxygen. Acc Chem Res 1995;28:141-5.

[86] Mukherjee S, Zhou L, Goodman AM, et al. Hot-electron-induced dissociation of $\mathrm{H} 2$ on gold nanoparticles supported on SiO2. J Am Chem Soc 2014;136:64-7.

[87] Mukherjee S, Libisch F, Large N, et al. Hot electrons do the impossible, plasmon-induced dissociation of $\mathrm{H} 2$ on $\mathrm{Au}$. Nano Lett 2013;13:240-7.

[88] Zhu H, Ke X, Yang X, Sarina S, Liu H. Reduction of nitroaromatic compounds on supported gold nanoparticles by visible and ultraviolet light. Angew Chem Int Ed 2010;49:9657-61.

[89] Guo J, Suástegui M, Sakimoto KK, et al. Light-driven fine chemical production in yeast biohybrids. Science 2018;362:813-6. 
[90] Kornienko N, Sakimoto KK, Herlihy DM, et al. Spectroscopic elucidation of energy transfer in hybrid inorganic-biological organisms for solar-to-chemical production. Proc Natl Acad Sci 2016;113:11750-5.

[91] Radziuk D, Moehwald H. Prospects for plasmonic hot spots in single molecule SERS towards the chemical imaging of live cells. Phys Chem Chem Phys 2015;17:21072-93.

[92] Kuznetsov Al, Miroshnichenko AE, Brongersma ML, Kivshar YS, Luk'yanchuk B. Optically resonant dielectric nanostructures. Science. 2016;354:aag2472.

[93] Liu GL, Long Y-T, Choi Y, Kang T, Lee LP. Quantized plasmon quenching dips nanospectroscopy via plasmon resonance energy transfer. Nat Methods 2007;4:1015-7.

[94] Zhou W, Gao X, Liu D, Chen X. Gold nanoparticles for in vitro diagnostics. Chem Rev 2015;115:10575-636.

[95] Dulkeith E, Morteani AC, Niedereichholz T, et al. Fluorescence quenching of dye molecules near gold nanoparticles, radiative and nonradiative effects. Phys Rev Lett 2002;89:203002.

[96] Bao Z, Liu X, Liu Y, Liu H, Zhao K. Near-infrared light-responsive inorganic nanomaterials for photothermal therapy. Asian J Pharm Sci 2016;11:349-64.

[97] Elahi N, Kamali M, Baghersad MH. Recent biomedical applications of gold nanoparticles: a review. Talanta 2018;184:537-56.

[98] Gharib M, Khalaf M, Afroz S, Feliu N, Parak W], Chakraborty I. Sustainable synthesis and improved colloidal stability of popcorn-shaped gold nanoparticles. ACS Sustain Chem Eng 2019;7:9834-41.

[99] Kim H, Chung K, Lee S, Kim DH, Lee H. Near-infrared lightresponsive nanomaterials for cancer theranostics. Wiley Interdiscip Rev Nanomed Nanobiotechnol 2016;8:23-45.

[100] Wei W, Zhang X, Zhang S, Wei G, Su Z. Biomedical and bioactive engineered nanomaterials for targeted tumor photothermal therapy, a review. Mater Sci Eng C 2019;104:109891.

[101] Chen C-W, Lee P-H, Chan Y-C, et al. Plasmon-induced hyperthermia, hybrid upconversion NaYF4:Yb/Er and gold nanomaterials for oral cancer photothermal therapy. J Mater Chem B 2015;3:8293-302.

[102] Dykman LA, Khlebtsov NG. Gold nanoparticles in chemo-, immuno-, and combined therapy, review [Invited]. Biomed Opt Express 2019;10:3152-82.

[103] Sreejivungsa K, Suchaichit N, Moosophon P, Chompoosor A. Light-regulated release of entrapped drugs from photoresponsive gold nanoparticles. J Nanomater 2016;2016:4964693.

[104] Wang D, Xu Z, Yu H, et al. Treatment of metastatic breast cancer by combination of chemotherapy and photothermal ablation using doxorubicin-loaded DNA wrapped gold nanorods. Biomaterials 2014;35:8374-84.

[105] Li W, Zhang X, Zhou M, et al. Functional core/shell drug nanoparticles for highly effective synergistic cancer therapy. Adv Healthc Mater 2014;3:1475-85.

[106] Park J, Park J, Ju EJ, et al. Multifunctional hollow gold nanoparticles designed for triple combination therapy and CT imaging. J Control Release 2015;207:77-85.

[107] Cheng Y, Samia AC, Meyers JD, Panagopoulos I, Fei B, Burda C. Highly efficient drug delivery with gold nanoparticle vectors for in vivo photodynamic therapy of cancer. J Am Chem Soc 2008;130:10643-7.
[108] Zhang D, Wu T, Qin X, et al. Intracellularly generated immunological gold nanoparticles for combinatorial photothermal therapy and immunotherapy against tumor. Nano Lett 2019;19:6635-46.

[109] Xiong R, Drullion C, Verstraelen P, et al. Fast spatial-selective delivery into live cells. J Control Release 2017;266:198-204.

[110] Wei L, Lu J, Xu H, Patel A, Chen Z-S, Chen G. Silver nanoparticles, synthesis, properties, and therapeutic applications. Drug Discov Today 2015;20:595-601.

[111] Wu P, Gao Y, Lu Y, Zhang H, Cai C. High specific detection and near-infrared photothermal therapy of lung cancer cells with high SERS active aptamer-silver-gold shell-core nanostructures. Analyst 2013;138:6501-10.

[112] Shi J, Wang L, Zhang J, et al. A tumor-targeting near-infrared laser-triggered drug delivery system based on GO@Ag nanoparticles for chemo-photothermal therapy and X-ray imaging. Biomaterials 2014;35:5847-61.

[113] Yu X, Liu W, Deng X, Yan S, Su Z. Gold nanocluster embedded bovine serum albumin nanofibers-graphene hybrid membranes for the efficient detection and separation of mercury ion. Chem Eng J 2018;335:176-84.

[114] Cui Y, Yang J, Zhou Q, et al. Renal clearable ag nanodots for in vivo computer tomography imaging and photothermal therapy. ACS Appl Mater Interfaces 2017;9:5900-6.

[115] Vankayala R, Sagadevan A, Vijayaraghavan P, Kuo C-L, Hwang KC. Metal nanoparticles sensitize the formation of singlet oxygen. Angew Chem Int Ed 2011;50:10640-4.

[116] Espinosa A, Curcio A, Cabana S, et al. Intracellular biodegradation of Ag nanoparticles, storage in ferritin, and protection by a Au shell for enhanced photothermal therapy. ACS Nano 2018;12:6523-35.

[117] Brandon MP, Ledwith DM, Kelly JM. Preparation of salinestable, silica-coated triangular silver nanoplates of use for optical sensing. J Colloid Interface Sci 2014;415:77-84.

[118] Palai PK, Mondal A, Chakraborti CK, Banerjee I, Pal K. Green synthesized amino-PEGylated silver decorated graphene nanoplatform as a tumor-targeted controlled drug delivery system. SN Appl Sci 2019;1:269.

[119] Jiang X, Zeng Q, Yu A. Thiol-frozen shape evolution of triangular silver nanoplates. Langmuir 2007;23:2218-23.

[120] Goodman AM, Cao Y, Urban C, et al. The surprising in vivo instability of near-IR-absorbing hollow Au-Ag nanoshells. ACS Nano 2014;8:3222-31.

[121] Wang L, Chen Y, Lin HY, et al. Near-IR-absorbing gold nanoframes with enhanced physiological stability and improved biocompatibility for in vivo biomedical applications. ACS Appl Mater Interfaces 2017;9:3873-84.

[122] Krasnok A, Caldarola M, Bonod N, Alú A. Spectroscopy and biosensing with optically resonant dielectric nanostructures. Adv Opt Mater 2018;6:1701094.

[123] Zyuzin MV, Baranov DG, Escudero A, et al. Photoluminescence quenching of dye molecules near a resonant silicon nanoparticle. Sci Rep 2018;8:1-7.

[124] Makarov SV, Petrov MI, Zywietz U, et al. Efficient secondharmonic generation in nanocrystalline silicon nanoparticles. Nano Lett 2017;17:3047-53.

[125] Xia B, Wang B, Chen Z, Zhang Q, Shi J. Near-infrared light-triggered intracellular delivery of anticancer drugs using porous silicon nanoparticles conjugated with IR820 dyes. Adv Mater Interfaces 2016;3:1500715. 
[126] Hong C, Lee J, Zheng H, Hong S-S, Lee C. Porous silicon nanoparticles for cancer photothermotherapy. Nanoscale Res Lett 2011;6:321.

[127] Dougherty TJ, Gomer CJ, Henderson BW, et al. Photodynamic therapy. JNCI J Natl Cancer Inst 1998;90:889-905.

[128] Kovalev D, Fujii M. Silicon nanocrystals, photosensitizers for oxygen molecules. Adv Mater 2005;17:2531-44.

[129] Xiao L, Gu L, Howell SB, Sailor MJ. Porous silicon nanoparticle photosensitizers for singlet oxygen and their phototoxicity against cancer cells. ACS Nano 2011;5:3651-9.

[130] Secret E, Maynadier M, Gallud A, et al. Two-photon excitation of porphyrin-functionalized porous silicon nanoparticles for photodynamic therapy. Adv Mater 2014;26:7643-8.

[131] Knežević NŽ, Stojanovic V, Chaix A, et al. Ruthenium(II) complex-photosensitized multifunctionalized porous silicon nanoparticles for two-photon near-infrared light responsive imaging and photodynamic cancer therapy. J Mater Chem B 2016;4:1337-42.

[132] Wang R, Zhao M, Deng D, et al. An intelligent and biocompatible photosensitizer conjugated silicon quantum dots- $\mathrm{MnO} 2$ nanosystem for fluorescence imaging-guided efficient photodynamic therapy. J Mater Chem B 2018;6:4592-601.

[133] Yin ZF, Wu L, Yang HG, Su YH. Recent progress in biomedical applications of titanium dioxide. Phys Chem Chem Phys 2013;15:4844-58.

[134] Ramakrishnan VM, Natarajan M, Santhanam A, Asokan V, Velauthapillai D. Size controlled synthesis of $\mathrm{TiO}_{2}$ nanoparticles by modified solvothermal method towards effective photo catalytic and photovoltaic applications. Mater Res Bull 2018;97:351-60.

[135] Liu W, Su P, Chen S, et al. Antibacterial and osteogenic stem cell differentiation properties of photoinduced $\mathrm{TiO}_{2}$ nanoparticle-decorated $\mathrm{TiO}_{2}$ nanotubes. Nanomed 2015;10:713-23.

[136] Yadav HM, Thorat ND, Yallapu MM, Tofail SAM, Kim J-S. Functional $\mathrm{TiO}_{2}$ nanocoral architecture for light-activated cancer chemotherapy. J Mater Chem B 2017;5:1461-70.

[137] Yang C-C, Sun Y-J, Chung P-H, et al. Development of Ce-doped $\mathrm{TIO}_{2}$ activated by $\mathrm{X}$-ray irradiation for alternative cancer treatment. Ceram Int 2017;43:12675-83.

[138] Nakayama M, Sasaki R, Ogino C, et al. Titanium peroxide nanoparticles enhanced cytotoxic effects of $\mathrm{X}$-ray irradiation against pancreatic cancer model through reactive oxygen species generation in vitro and in vivo. Radiat Oncol 2016;11:91.

[139] Antonarakis ES, Emadi A. Ruthenium-based chemotherapeutics, are they ready for prime time? Cancer Chemother Pharmacol 2010;66:1-9.

[140] Nešić M, Žakula J, Korićanac L, et al. Light controlled metallodrug delivery system based on the $\mathrm{TiO}_{2}$-nanoparticles and Ru-complex. J Photochem Photobiol Chem 2017;347:55-66.

[141] Guo Z, Zheng K, Tan Z, et al. Overcoming drug resistance with functional mesoporous titanium dioxide nanoparticles combining targeting, drug delivery and photodynamic therapy. J Mater Chem B 2018;6:7750-9.

[142] Escudero A, Carrillo-Carrión C, Zyuzin MV, et al. Synthesis and functionalization of monodisperse near-ultraviolet and visible excitable multifunctional Eu 3+, Bi $3+$, REVO 4 nanophosphors for bioimaging and biosensing applications. Nanoscale 2016;8:12221-36.

[143] Escudero A, Carrillo-Carrión C, Zyuzin MV, Parak WJ. Luminescent rare-earth-based nanoparticles: a summarized overview of their synthesis, functionalization, and applications. Top Curr Chem 2016;374:48.

[144] Escudero A, Becerro Al, Carrillo-Carrión C, et al. Rare earth based nanostructured materials: synthesis, functionalization, properties and bioimaging and biosensing applications. Nanophotonics 2017;6:881-921.

[145] Szacitowski K, Macyk W, Drzewiecka-Matuszek A, Brindell $M$, Stochel G. Bioinorganic photochemistry: frontiers and mechanisms. Chem Rev 2005;105:2647-94.

[146] Yin M, Ju E, Chen Z, Li Z, Ren J, Qu X. Upconverting nanoparticles with a mesoporous $\mathrm{TIO}_{2}$ shell for near-infrared-triggered drug delivery and synergistic targeted cancer therapy. Chem - Eur J 2014;20:14012-7.

[147] Zhang H, Zhang H, Zhu X, et al. Visible-light-sensitive titanium dioxide nanoplatform for tumor-responsive Fe2 + liberating and artemisinin delivery. Oncotarget 2017;8:58738-53.

[148] Espinosa A, Di Corato R, Kolosnjaj-Tabi J, Flaud P, Pellegrino $\mathrm{T}$, Wilhelm C. Duality of iron oxide nanoparticles in cancer therapy, amplification of heating efficiency by magnetic hyperthermia and photothermal bimodal treatment. ACS Nano 2016;10:2436-46.

[149] Bigall NC, Dilena E, Dorfs D, et al. Hollow iron oxide nanoparticles in polymer nanobeads as MRI contrast agents. J Phys Chem C 2015;119:6246-53.

[150] Espinosa A, Kolosnjaj-Tabi J, Abou-Hassan A, et al. Magnetic (Hyper)thermia or photothermia? Progressive comparison of iron oxide and gold nanoparticles heating in water, in cells, and in vivo. Adv Funct Mater 2018;28:1803660.

[151] Zyuzin MV, Cassani M, Barthel MJ, et al. Confining iron oxide nanocubes inside submicrometric cavities as a key strategy to preserve magnetic heat losses in an intracellular environment. Appl Mater Interfaces 2019;11:41957-71.

[152] Chen H, Burnett J, Zhang F, Zhang J, Paholak H, Sun D. Highly crystallized iron oxide nanoparticles as effective and biodegradable mediators for photothermal cancer therapy. J Mater Chem B 2014;2:757-65.

[153] He YP, Miao YM, Li CR, et al. Size and structure effect on optical transitions of iron oxide nanocrystals. Phys Rev B 2005;71:125411.

[154] Zhao Y, Sadat ME, Dunn A, et al. Photothermal effect on Fe304 nanoparticles irradiated by white-light for energyefficient window applications. Sol Energy Mater Sol Cells 2017;161:247-54.

[155] Oh Y, Je J-Y, Moorthy MS, Seo H, Cho WH. pH and NIRlight-responsive magnetic iron oxide nanoparticles for mitochondria-mediated apoptotic cell death induced by chemo-photothermal therapy. Int J Pharm 2017;531:1-13.

[156] Shen S, Ding B, Zhang S, et al. Near-infrared light-responsive nanoparticles with thermosensitive yolk-shell structure for multimodal imaging and chemo-photothermal therapy of tumor. Nanomedicine Nanotechnol Biol Med 2017;13: 1607-16.

[157] Wang H, Mu Q, Revia R, et al. Iron oxide-carbon core-shell nanoparticles for dual-modal imaging-guided photothermal therapy. J Control Release 2018;289:70-8.

[158] Wang D, Zhu L, Chen J-F, Dai L. Liquid marbles based on magnetic upconversion nanoparticles as magnetically and optically responsive miniature reactors for photocatalysis and photodynamic therapy. Angew Chem Int Ed 2016;55:10795-9. 
[159] Bagheri A, Arandiyan H, Boyer C, Lim M. Lanthanide-doped upconversion nanoparticles, emerging intelligent light-activated drug delivery systems. Adv Sci 2016;3:1500437.

[160] Yao C, Wang P, Li X, et al. Near-infrared-triggered azobenzeneliposome/upconversion nanoparticle hybrid vesicles for remotely controlled drug delivery to overcome cancer multidrug resistance. Adv Mater 2016;28:9341-8.

[161] Fedoryshin LL, Tavares AJ, Petryayeva E, Doughan S, Krull UJ. Near-infrared-triggered anticancer drug release from upconverting nanoparticles. ACS Appl Mater Interfaces 2014;6:13600-6.

[162] Xing Q, Li N, Jiao Y, et al. Near-infrared light-controlled drug release and cancer therapy with polymer-caged upconversion nanoparticles. RSC Adv 2015;5:5269-76.

[163] Jalani G, Naccache R, Rosenzweig DH, Haglund L, Vetrone F, Cerruti M. Photocleavable hydrogel-coated upconverting nanoparticles, a multifunctional theranostic platform for NIR imaging and on-demand macromolecular delivery. J Am Chem Soc 2016;138:1078-83.

[164] Tian G, Zheng X, Zhang X, et al. TPGS-stabilized NaYbF4,Er upconversion nanoparticles for dual-modal fluorescent/CT imaging and anticancer drug delivery to overcome multi-drug resistance. Biomaterials 2015;40:107-16.

[165] Feliu N, Hühn J, Zyuzin MV, et al. Quantitative uptake of colloidal particles by cell cultures. Sci Total Environ 2016;568:819-28.

[166] Xing P, Zhao Y. multifunctional nanoparticles self-assembled from small organic building blocks for biomedicine. Adv Mater 2016;28:7304-39.

[167] Mitragotri S, Stayton P. Organic nanoparticles for drug delivery and imaging. MRS Bull 2014;39:219-23.

[168] Huang Y, Dong R, Zhu X, Yan D. Photo-responsive polymeric micelles. Soft Matter 2014;10:6121-38.

[169] Kotharangannagari VK, Sánchez-Ferrer A, Ruokolainen J, Mezzenga R. Photoresponsive reversible aggregation and dissolution of rod-coil polypeptide diblock copolymers. Macromolecules 2011;44:4569-73.

[170] Molla MR, Rangadurai P, Antony L, Swaminathan S, de Pablo JJ, Thayumanavan S. Dynamic actuation of glassy polymersomes through isomerization of a single azobenzene unit at the block copolymer interface. Nat Chem 2018;10:659-66.

[171] Yuan X, Wang Z, Li L, et al. Novel fluorescent amphiphilic copolymer probes containing azo-tetraphenylethylene bridges for azoreductase-triggered release. Mater Chem Front 2019;3:1097-104.

[172] Li Q, Cao Z, Wang G. Diazonaphthoquinone-based amphiphilic polymer assemblies for NIR/UV light- and $\mathrm{pH}$-responsive controlled release. Polym Chem 2018;9:463-71.

[173] Ji S, Xu L, Fu X, Sun J, Li Z. Light- and metal ion-induced self-assembly and reassembly based on block copolymers containing a photoresponsive polypeptide segment. Macromolecules 2019;52:4686-93.

[174] Gebeyehu BT, Huang S-Y, Lee A-W, et al. Dual stimuli-responsive nucleobase-functionalized polymeric systems as efficient tools for manipulating micellar self-assembly behavior. Macromolecules 2018;51:1189-97.

[175] Ma Y, Liang X, Tong S, Bao G, Ren Q, Dai Z. Gold nanoshell nanomicelles for potential magnetic resonance imaging, lighttriggered drug release, and photothermal therapy. Adv Funct Mater 2013;23:815-22.
[176] Linsley CS, Wu BM. Recent advances in light-responsive ondemand drug-delivery systems. Ther Deliv 2017;8:89-107.

[177] Lee Y, Thompson DH. Stimuli-responsive liposomes for drug delivery. Wiley Interdiscip Rev Nanomed Nanobiotechnol 2017;9:e1450.

[178] Pattni BS, Chupin VV, Torchilin VP. New developments in liposomal drug delivery. Chem Rev 2015;115:10938-66.

[179] Daraee H, Etemadi A, Kouhi M, Alimirzalu S, Akbarzadeh A. Application of liposomes in medicine and drug delivery. Artif Cells Nanomedicine Biotechnol 2016;44:381-91.

[180] Wu G, Mikhailovsky A, Khant HA, Zasadzinski JA. Synthesis, characterization, and optical response of gold nanoshells used to trigger release from liposomes. Meth Enzymol 2009;464:279-307.

[181] Kojima C, Hirano Y, Kono K. Preparation of complexes of liposomes with gold nanoparticles. Meth Enzymol 2009; 464:131-45

[182] Palankar R, Pinchasik B-E, Khlebtsov BN, et al. Nanoplasmonically-induced defects in lipid membrane monitored by ion current, transient nanopores versus membrane rupture. Nano Lett 2014;14:4273-9.

[183] Luo L, Bian Y, Liu Y, et al. Combined near infrared photothermal therapy and chemotherapy using gold nanoshells coated liposomes to enhance antitumor effect. Small 2016;12:4103-12.

[184] Lajunen T, Viitala L, Kontturi L-S, et al. Light induced cytosolic drug delivery from liposomes with gold nanoparticles. J Control Release 2015;203:85-98.

[185] Li Q, Tang Q, Zhang P, et al. Human epidermal growth factor receptor 2 antibodies enhance the specificity and anticancer activity of light-sensitive doxorubicin-labeled liposomes. Biomaterials 2015;57:1-11.

[186] Wiraja C, Mathiyazhakan M, Movahedi F, et al. Near-infrared light-sensitive liposomes for enhanced plasmid DNA transfection. Bioeng Transl Med 2016;1:357-64.

[187] Li Q, Li W, Di H, et al. A photosensitive liposome with NIR light triggered doxorubicin release as a combined photodynamic-chemo therapy system. J Control Release 2018;277:114-25.

[188] Sukhorukov GB, Donath E, Lichtenfeld H, et al. Layer-by-layer self assembly of polyelectrolytes on colloidal particles. Colloids Surf Physicochem Eng Asp 1998;137:253-66.

[189] Timin AS, Peltek O0, Zyuzin MV, et al. Safe and effective delivery of antitumor drug using mesenchymal stem cells impregnated with submicron carriers. ACS Appl Mater Interfaces 2019;11:13091-104.

[190] Timin AS, Muslimov AR, Lepik KV, et al. Efficient gene editing via non-viral delivery of CRISPR-Cas9 system using polymeric and hybrid microcarriers. Nanomedicine Nanotechnol Biol Med 2018;14:97-108.

[191] Rivera_Gil P, Nazarenus M, Ashraf S, Parak WJ. pH-sensitive capsules as intracellular optical reporters for monitoring lysosomal pH changes upon stimulation. Small 2012;8:943-8.

[192] Tu Y, Gu Y, Horn RMV, Mitrokhin M, Harris FW, Cheng SZD. A synthetic approach towards micron-sized smectic liquid crystal capsules via the diffusion controlled swelling method. Polym Chem 2015;6:2551-9.

[193] Marturano V, Cerruti P, Giamberini M, Tylkowski B, Ambrogi V. Light-responsive polymer micro- and nano-capsules. Polymers 2017;9:8. 
[194] Parakhonskiy BV, Parak WJ, Volodkin D, Skirtach AG. Hybrids of polymeric capsules, lipids, and nanoparticles, thermodynamics and temperature rise at the nanoscale and emerging applications. Langmuir 2019;35:8574-83.

[195] Geints YuE, Panina EK, Zemlyanov AA. Features of light absorption by an ensemble of microcapsules. Atmospheric Ocean Opt 2017;30:441-5.

[196] Carregal-Romero S, Ochs M, Rivera-Gil P, et al. NIR-light triggered delivery of macromolecules into the cytosol. J Control Release 2012;159:120-7.

[197] Skirtach AG, Muñoz Javier A, Kreft O, et al. Laser-induced release of encapsulated materials inside living cells. Angew Chem Int Ed 2006;45:4612-7.

[198] Baffou G. Thermoplasmonics heating metal nanoparticles using light. Cambridge, England: Cambridge University Press, 2017.

[199] Ambrosone A, Marchesano V, Carregal-Romero S, Intartaglia $D$, Parak WJ, Tortiglione C. Control of Wnt/ $\beta$-catenin signaling pathway in vivo via light responsive capsules. ACS Nano 2016;10:4828-34.

[200] Ott A, Yu X, Hartmann R, et al. Light-addressable and degradable silica capsules for delivery of molecular cargo to the cytosol of cells. Chem Mater 2015;27:1929-42.

[201] N’Gom M, Li S, Schatz G, et al. Electron-beam mapping of plasmon resonances in electromagnetically interacting gold nanorods. Phys Rev B 2009;80:113411.

[202] Timin AS, Muslimov AR, Zyuzin MV, et al. Multifunctional scaffolds with improved antimicrobial properties and osteogenicity based on piezoelectric electrospun fibers decorated with bioactive composite microcapsules. ACS Appl Mater Interfaces 2018;10:34849-68.

[203] Shao J, Xuan M, Si T, Dai L, He Q. Biointerfacing polymeric microcapsules for in vivo near-infrared light-triggered drug release. Nanoscale 2015;7:19092-8.

[204] Lengert E, Parakhonskiy B, Khalenkow D, et al. Laser-induced remote release in vivo in $C$. elegans from novel silver nanoparticles-alginate hydrogel shells. Nanoscale 2018;10:17249-56.

[205] Wu Z, Gao C, Frueh J, Sun J, He Q. Remote-controllable explosive polymer multilayer tubes for rapid cancer cell killing. Macromol Rapid Commun 2015;36:1444-9.

[206] Kurapati R, Raichur AM. Near-infrared light-responsive graphene oxide composite multilayer capsules, a novel route for remote controlled drug delivery. Chem Commun 2012;49:734-6.

[207] Yashchenok A, Masic A, Gorin D, et al. Optical heating and temperature determination of core-shell gold nanoparticles and single-walled carbon nanotube microparticles. Small 2015;11:1320-7.

[208] Zhang Z, Taylor M, Collins C, et al. Light-activatable theranostic agents for image-monitored controlled drug delivery. ACS Appl Mater Interfaces 2018;10:1534-43.

[209] Xiong R, Soenen SJ, Braeckmans K, Skirtach AG. Towards theranostic multicompartment microcapsules, in-situ diagnostics and laser-induced treatment. Theranostics 2013;3:141-51.

[210] Park K. Facing the truth about nanotechnology in drug delivery. ACS Nano 2013;7:7442-7.

[211] Corbo C, Molinaro R, Taraballi F, et al. Unveiling the in vivo protein corona of circulating leukocyte-like carriers. ACS Nano 2017;11:3262-73.
[212] Neubi GMN, Opoku-Damoah Y, Gu X, Han Y, Zhou J, Ding Y. Bio-inspired drug delivery systems, an emerging platform for targeted cancer therapy. Biomater Sci 2018;6:958-73.

[213] Kim MW, Kwon S-H, Choi JH, Lee A. A promising biocompatible platform, lipid-based and bio-inspired smart drug delivery systems for cancer therapy. Int J Mol Sci 2018;19:3859.

[214] Chen Z, Hu Q, Gu Z. Leveraging engineering of cells for drug delivery. Acc Chem Res 2018;51:668-77.

[215] Timin AS, Litvak MM, Gorin DA, Atochina-Vasserman EN, Atochin DN, Sukhorukov GB. Cell-based drug delivery and use of nano-and microcarriers for cell functionalization. Adv Healthc Mater 2018;7:1700818.

[216] Parayath NN, Amiji MM. Therapeutic targeting strategies using endogenous cells and proteins. J Control Release 2017;258:81-94.

[217] Orbach A, Zelig O, Yedgar S, Barshtein G. Biophysical and biochemical markers of red blood cell fragility. Transfus Med Hemotherapy 2017;44:183-7.

[218] Han X, Wang C, Liu Z. Red blood cells as smart delivery systems. Bioconjug Chem 2018;29:852-60.

[219] Delcea M, Sternberg N, Yashchenok AM, et al. Nanoplasmonics for dual-molecule release through nanopores in the membrane of red blood cells. ACS Nano 2012;6:4169-80.

[220] Gao M, Hu A, Sun X, et al. Photosensitizer decorated red blood cells as an ultrasensitive light-responsive drug delivery system. ACS Appl Mater Interfaces 2017;9:5855-63.

[221] Villa CH, Cines DB, Siegel DL, Muzykantov V. Erythrocytes as carriers for drug delivery in blood transfusion and beyond. Transfus Med Rev 2017;31:26-35.

[222] Banks WA. The blood-brain barrier in neuroimmunology, tales of separation and assimilation. Brain Behav Immun 2015;44:1-8.

[223] Xie Z, Su Y, Kim GB, et al. Immune cell-mediated biodegradable theranostic nanoparticles for melanoma targeting and drug delivery. Small. 2017;13:1603121.

[224] Prinyakupt J, Pluempitiwiriyawej C. Segmentation of white blood cells and comparison of cell morphology by linear and naïve Bayes classifiers. Biomed Eng OnLine 2015;14:63.

[225] Madsen SJ, Baek S-K, Makkouk AR, Krasieva T, Hirschberg H. Macrophages as cell-based delivery systems for nanoshells in photothermal therapy. Ann Biomed Eng 2012;40:507-15.

[226] Zhang C, Zhang J, Shi G, et al. A light responsive nanoparticlebased delivery system using pheophorbide a graft polyethylenimine for dendritic cell-based cancer immunotherapy. Mol Pharm 2017;14:1760-70.

[227] Chabot-Richards DS, George TI. White blood cell counts, reference methodology. Clin Lab Med 2015;35:11-24.

[228] Su Y, Xie Z, Kim GB, Dong C, Yang J. Design strategies and applications of circulating cell-mediated drug delivery systems. ACS Biomater Sci Eng 2015;1:201-17.

[229] Chu D, Dong X, Zhao Q, Gu J, Wang Z. Photosensitization priming of tumor microenvironments improves delivery of nanotherapeutics via neutrophil infiltration. Adv Mater 2017;29:1701021.

[230] Ogiya R, Niikura N, Kumaki N, et al. Comparison of tumor-infiltrating lymphocytes between primary and metastatic tumors in breast cancer patients. Cancer Sci 2016;107:1730-5.

[231] Becker PSA, Suck G, Nowakowska P, et al. Selection and expansion of natural killer cells for NK cell-based immunotherapy. Cancer Immunol Immunother 2016;65:477-84. 
[232] Deng G, Sun Z, Li S, et al. Cell-membrane immunotherapy based on natural killer cell membrane coated nanoparticles for the effective inhibition of primary and abscopal tumor growth. ACS Nano 2018;12:12096-108.

[233] Snyder RJ, Lantis J, Kirsner RS, Shah V, Molyneaux M, Carter MJ. Macrophages. A review of their role in wound healing and their therapeutic use. Wound Repair Regen 2016;24:613-29.

[234] Choi M-R, Stanton-Maxey KJ, Stanley JK, et al. A cellular trojan horse for delivery of therapeutic nanoparticles into tumors. Nano Lett 2007;7:3759-65.

[235] Klyachko NL, Polak R, Haney MJ, et al. Macrophages with cellular backpacks for targeted drug delivery to the brain. Biomaterials 2017;140:79-87.

[236] Krueger TEG, Thorek DLJ, Denmeade SR, Isaacs JT, Brennen WN. Concise review, mesenchymal stem cell-based drug delivery, the good, the bad, the ugly, and the promise. STEM CELLS Transl Med 2018;7:651-63.

[237] Xia J, Tsai A-C, Cheng W, Yuan X, Ma T, Guan J. Development of a microdevice-based human mesenchymal stem cell-mediated drug delivery system. Biomater Sci 2019;7:2348-57.

[238] Mar Encabo-Berzosa M, Gimeno M, Lujan L, et al. Selective delivery of photothermal nanoparticles to tumors using mesenchymal stem cells as Trojan horses. RSC Adv 2016;6:58723-32.

[239] Quaranta P, Focosi D, Freer G, Pistello M. Tweaking mesenchymal stem/progenitor cell immunomodulatory properties with viral vectors delivering cytokines. Stem Cells Dev 2016;25:1321-41.

[240] Xu C, Feng Q, Yang H, et al. A light-triggered mesenchymal stem cell delivery system for photoacoustic imaging and chemo-photothermal therapy of triple negative breast cancer. Adv Sci 2018;5:1800382.

[241] Muslimov AR, Timin AS, Bichaykina VR, et al. Biomimetic drug delivery platforms based on mesenchymal stem cells impregnated with light-responsive submicron sized carriers. Biomater Sci 2020. DOI: 10.1039/C9BM00926D.

[242] Qian C, Feng P, Yu J, et al. Innentitelbild, anaerobe-inspired anticancer nanovesicles (Angew. Chem. 10/2017). Angew Chem 2017;129:2558.

[243] Hosseinidoust Z, Mostaghaci B, Yasa O, Park B-W, Singh AV, Sitti $M$. Bioengineered and biohybrid bacteria-based systems for drug delivery. Adv Drug Deliv Rev 2016;106:27-44.

[244] Pastrana E. Optogenetics, controlling cell function with light. Nat Methods 2011;8:24-5.

[245] Motta-Mena LB, Reade A, Mallory MJ, et al. An optogenetic gene expression system with rapid activation and deactivation kinetics. Nat Chem Biol 2014;10:196-202.
[246] Xie S, Chen M, Song X, et al. Bacterial microbots for acidlabile release of hybrid micelles to promote the synergistic antitumor efficacy. Acta Biomater 2018;78:198-210.

[247] Somiya M, Liu Q, Kuroda S. Current progress of virusmimicking nanocarriers for drug delivery. Nanotheranostics 2017;1:415-29.

[248] Luo C-H, Huang C-T, Su C-H, Yeh C-S. Bacteria-mediated hypoxia-specific delivery of nanoparticles for tumors imaging and therapy. Nano Lett 2016;16:3493-9.

[249] Hajam IA, Dar PA, Appavoo E, Kishore S, Bhanuprakash V, Ganesh K. Bacterial ghosts of escherichia coli drive efficient maturation of bovine monocyte-derived dendritic cells. PLoS One 2015;10:e0144397.

[250] Hajam IA, Dar PA, Won G, Lee JH. Bacterial ghosts as adjuvants: mechanisms and potential. Vet Res 2017;48:37.

[251] Thang DC, Wang Z, Lu X, Xing B. Precise cell behaviors manipulation through light-responsive nano-regulators: recent advance and perspective. Theranostics 2019;9:3308-40.

[252] Gujrati V, Prakash J, Malekzadeh-Najafabadi J, et al. Bioengineered bacterial vesicles as biological nano-heaters for optoacoustic imaging. Nat Commun 2019;10:1-10.

[253] Bouard D, Alazard-Dany N, Cosset F-L. Viral vectors: from virology to transgene expression. Br J Pharmacol 2009;157:153-65.

[254] Kotterman MA, Chalberg TW, Schaffer DV. Viral vectors for gene therapy: translational and clinical outlook. Annu Rev Biomed Eng 2015;17:63-89.

[255] Everts M, Saini V, Leddon JL, et al. Covalently linked au nanoparticles to a viral vector: potential for combined photothermal and gene cancer therapy. Nano Lett 2006;6:587-91.

[256] Jung B-K, Lee YK, Hong J, Ghandehari H, Yun C-O. Mild hyperthermia induced by gold nanorod-mediated plasmonic photothermal therapy enhances transduction and replication of oncolytic adenoviral gene delivery. ACS Nano 2016;10:10533-43.

[257] Pandori MW, Sano T. Photoactivatable retroviral vectors, a strategy for targeted gene delivery. Gene Ther 2000;7: 1999-2006.

[258] Gilchrist RK, Medal R, Shorey WD, Hanselman RC, Parrott JC, Taylor CB. Selective inductive heating of lymph nodes. Ann Surg 1957;146:596-606.

[259] Johannsen M, Gneveckow U, Eckelt L, et al. Clinical hyperthermia of prostate cancer using magnetic nanoparticles, Presentation of a new interstitial technique. Int J Hyperthermia 2005;21:637-47. 